\title{
ON EXPLICIT CONNECTIONS BETWEEN DYNAMICAL AND PARAMETER SPACES
}

\author{
Genadi LEVIN
}

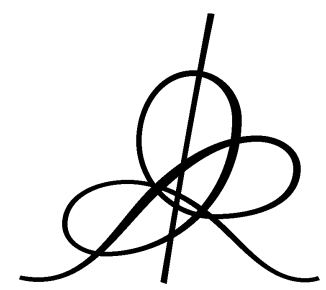

Institut des Hautes Études Scientifiques

35 , route de Chartres

91440 - Bures-sur-Yvette (France)

Février 2002

IHES/M/02/05 


\title{
On explicit connections between dynamical and parameter spaces
}

\author{
Genadi Levin * \\ Inst. of Math., Hebrew University, Jerusalem 91904, Israel
}

\begin{abstract}
Studying holomorphic dynamical systems, we find some new relations between dynamical and parameter spaces, in particular, between velocity of motions in the parameter space and areas in dynamical ones. Applications to the Mandelbrot sets are given.
\end{abstract}

\section{Contents}

1. Main results.

2. Theorem 2 and its corollaries.

3. Proof of Theorem 1 for H-components.

4. Non-hyperbolic components.

5. Example 1.

6. On parabolic maps.

7. Generalizations to polynomial-like maps.

\section{Main results}

Since [5], [6], the idea of connections between dynamical and parameter spaces plays one of the central roles in modern holomorphic dynamics. For example, it is used in [5] to prove the connectivity of the Mandelbrot set.

\subsection{An inequality and examples}

Let $M_{\ell}$ be the Mandelbrot set of the family $f_{c}: z \mapsto z^{\ell}+c$, where $\ell \geq 2$, i.e. the set of $c \in \mathbf{C}$ such that the Julia set $J_{c}$ of $f_{c}$ is connected. For every $\ell$, it is a connected compact in the plane [5], [6]. Let $W$ be a component of the interior

\footnotetext{
${ }^{*}$ Supported in part by IMPAN-BC European Community Centre of Excellence
} 
$\operatorname{int}\left(M_{\ell}\right)$ of $M_{\ell}$. By the maximal principle, $W$ is simply-connected. A priori, $W$ is of one of the following two types:

- H-component, i.e. hyperbolic one: for every $c \in W$, the map $f_{c}$ has an attracting periodic orbit $P(c)=\left\{a_{1}(c), \ldots, a_{p}(c)\right\}$ of period $p=p(W)$, so that the period $p$ is fixed throughout $W$, and every $a_{j}(c)$ is a holomorphic function on $c \in W$. Moreover, the Julia set $J_{c}$ of $f_{c}$ has zero Lebesgue measure (area), and the union of the bounded components of $\mathbf{C} \backslash J_{c}$, which we will call the (bounded) Fatou set $F_{c}$ of $f_{c}$, coincides with the basin of attraction of $P(c)$.

- N-component, i.e. non-hyperbolic one: it can be characterized so that, for every $c \in W$, the Julia set $J_{c}$ of $f_{c}$ has a positive Lebesgue measure (area), [14]. The Fatou set $F_{c}$ is empty.

The Fatou, or Density of Hyperbolicity Conjecture says that N-components do not exist. Since it is an open problem, we consider N-components as well.

Denote by $K_{c}$ the filled-in Julia set of $f_{c}$, i.e. $K_{c}=J_{c} \cup F_{c}$. In particular, if $c$ is in N-component, then $K_{c}=J_{c}$.

Denote by $\operatorname{dist}(z, A)$ the Euclidean distance between a point $z$ and a set $A$ of the plane.

At last, denote by $\sigma$ the Lebesgue measure on the plane, and by $\operatorname{area}(A)$ the Lebesgue measure of a subset $A$ of the plane.

Theorem 1 There exists an absolute constant $\mathbf{L}_{*}$, such that, for every $\ell \geq 2$ and every $c$ in the interior of $M_{\ell}$,

$$
\operatorname{dist}\left(c, \partial M_{\ell}\right) \leq \mathbf{L}_{*} \cdot \ell^{2} \int_{K_{c}}|z|^{\ell-2} d \sigma_{z} .
$$

Moreover,

(H) there exist absolute constants $\mathbf{L}_{H}>0$ and $a_{*}>0$ such that, for every $\ell \geq 2$, for every $H$-component $W$ of $M_{\ell}$, and every $c \in W$, if the multiplier $\rho$ of the periodic orbit $P(c)$ is such that $|\rho|<\ell a_{*}^{\ell}$, then

$$
\operatorname{dist}\left(c, \partial M_{\ell}\right) \leq \mathbf{L}_{H} \cdot \ell \int_{F_{c}}|z|^{\ell-2} d \sigma_{z} .
$$

(N) if $c$ is in an $N$-component of $M_{\ell}$, then

$$
\operatorname{dist}\left(c, \partial M_{\ell}\right) \leq \frac{4 \ell}{\pi} \int_{J_{c}}|z|^{\ell-2} d \sigma_{z} .
$$

For polynomial-like maps, see last Section. 
Comment 1 It is easy to check that if $c \in M_{\ell}$ and $z \in J_{c}$, then $|z| \leq 2^{\frac{1}{\ell-1}}$. Therefore,

$$
\int_{K_{c}}|z|^{\ell-2} d \sigma_{z}<2 \cdot \operatorname{area}\left(K_{c}\right)
$$

Comment 2 Let $c$ be in a hyperbolic component of $M_{\ell}$. Then 0 lies in a component $\Omega$ of $F_{c}, r:=\operatorname{dist}\left(0, J_{c}\right)>0$, and the "first approximation" of $\ell \int_{\Omega}|z|^{\ell-2} d \sigma_{z}$ is

$$
\ell \int_{|z|<r}|z|^{\ell-2} d \sigma_{z}=2 \pi r^{\ell}=2 \pi \operatorname{dist}\left(c, J_{c}\right)
$$

Comment 3 If $\rho=0$, then one can take $L_{H}=4 / \pi$.

Consider 3 examples.

Example 1. It is the following statement ${ }^{1}$, which will be proved in Section 5 . It shows that the inequality (1) is exact in the following sense:

For every Misiurewicz value of parameter $c_{*} \in M_{2}$ there is a constant $L$ and a sequence $c_{n}$, which converges to $c_{*}$, such that, for every $c_{n}, 0$ is a periodic point of $f_{c_{n}}$ and

$$
\frac{\operatorname{dist}\left(c_{n}, \partial M_{2}\right)}{\operatorname{area}\left(K_{c_{n}}\right)} \geq L
$$

and, moreover,

$$
L \leq \frac{\operatorname{dist}\left(c_{n}, \partial M_{2}\right)}{\operatorname{dist}\left(c_{n}, J_{c_{n}}\right)} \leq \frac{1}{L}
$$

In Examples 2, 3 below we use the parameter plane to get information about dynamical pictures.

Example 2. It shows that, in general, $\operatorname{dist}\left(c, \partial M_{\ell}\right)$ and $\operatorname{dist}\left(c, J_{c}\right)$ are not comparable. For the Mandelbrot set $M_{2}$, consider the Feigenbaum parameter $c^{F}=-1.4 \ldots$ and the sequence $c_{p}^{F}, p>0$, of real parameters which converges to the Feigenbaum parameter $c^{F}$, so that $c_{p}^{F}>c^{F}$ and 0 is a periodic point of the map $f_{c_{p}^{F}}$ of period $2^{p}, p=0,1, \ldots$. According to the Feigenbaum-Coullet-Tresser Universality [9],[3],[19],[17],[13], $\operatorname{dist}\left(c_{p}^{F}, J_{c_{p}^{F}}\right) \sim \operatorname{const} \alpha^{-2 p}$ while $\operatorname{dist}\left(c_{p}^{F}, \partial M_{2}\right)>$ const $\delta^{-p}$ so that $\left.\operatorname{dist}\left(c_{p}^{F}\right), \partial M_{2}\right) / \operatorname{dist}\left(c_{p}^{F}, J_{c_{p}^{F}}\right) \rightarrow \infty$ as $p \rightarrow \infty($ where $\delta=4.66 \ldots$ and $\alpha=-2.5 \ldots$ are two universal constants). The inequality ( 1$)$ then gives that $\operatorname{area}\left(K_{c_{p}^{F}}\right) \geq\left(1 / \mathbf{L}_{*}\right) \delta^{-p}$

\footnotetext{
${ }^{1}$ Jointly with F. Przytycki
} 
Example 3. Let $W_{p / q}$ be the hyperbolic component of $M_{2}$, which is tangent to the boundary of the main cardioid at the point $c$ where $f_{c}$ has a fixed point with multiplier $\exp (2 \pi i p / q)$. There is a conjecture saying that the "size" of $W_{p / q}$ (outside of a neighborhood of the cusp $c=1 / 4$ ) is of precise order $1 / q^{2}$. Then ( 1 ) would imply that $\operatorname{area}\left(K_{c_{p / q}}\right) \geq \mathrm{const} / q^{2}$, where $c_{p / q}$ is the center of $W_{p / q}$, and const does not depend on $p / q$.

\subsection{Velocity of motion of periodic orbits}

Let us fix the degree $\ell \geq 2$.

We use tools similar to those in [11], see also [15], [8]. Consider Ruelle transfer operator $T=T_{c}$ defined for the map $f=f_{c}$ as follows:

$$
T g(z)=\sum_{w: f(w)=z} \frac{g(w)}{\left(f^{\prime}(w)\right)^{2}}
$$

acting on functions $g$, wherever the right-hand side is defined.

It is related to so-called backward invariant Beltrami forms:

Definition 1.1 (cf. [14], [16]).

Let $f=f_{c}$, and $V$ be a measurable set, such that $f^{-1}(V) \subset V$. Backward invariant Beltrami form on the set $V$ is a function $\mu \in L_{\infty}(V)$, such that, for a.e. $x \in f^{-1}(V)$,

$$
\mu(f(x))=\mu(x) \cdot \frac{\left(f^{\prime}(x)\right)^{2}}{\left|f^{\prime}(x)\right|^{2}}
$$

Then $\mu$ like this is closely related to the operator $T$ via the following duality:

Fact 1.1 For every function $g$, if $\mu \cdot g$ is integrable on the set $V$, then

$$
\int_{V} \mu(z) T g(z) d \sigma_{z}=\int_{f^{-1}(V)} \mu(z) g(z) d \sigma_{z}
$$

Proof.

$$
\int_{f^{-1}(V)} \mu(w) g(w) d \sigma_{w}=\int_{V}\left(\sum_{f(w)=z} \frac{\mu(w) g(w)}{\left|f^{\prime}(w)\right|^{2}}\right) d \sigma_{z}=\int_{V} \mu(z) T g(z) d \sigma_{z} .
$$

If $\Omega$ is a component of an immediate basin of attraction of an attracting or parabolic periodic orbit of $f=f_{c}$, then we use the following construction of backward invariant Beltrami forms $\mu$. Let $C \subset \Omega$ be a fundamental region of $f$, i.e. every grand orbit of $f$ enters $C$ at most once. Now define

$$
V=V_{C}=\cup_{n \geq 0} f^{-n}(C) .
$$


Then choose an arbitrary $\mu \in L_{\infty}(C)$ and define extension of $\mu$ from $C$ to a backward invariant form on $V$ according to the equation (4).

A relation of all this to the parameter plane is described in the following theorem. Let $O=\left\{b_{k}\right\}_{k=1}^{n}$ be any periodic orbit $O=\left\{b_{k}\right\}_{k=1}^{n}$ of $f=f_{c}$ of exact period $n$ with the multiplier $\rho \neq 1$. First, since $\rho \neq 1$, there is a holomorphic extension of the periodic orbit $O=O(c)$ of $f_{c}$ to a periodic orbit $O(\hat{c})=\left\{b_{k}(\hat{c})\right\}_{k=1}^{n}$ of $f_{\hat{c}}$ for $\hat{c}$ in a neighborhood of $c$. In particular, if $\rho(\hat{c})$ denotes the multiplier of $O(\hat{c})$, it is holomorphic in $\hat{c}$ in this neighborhood. We denote by $b_{k}^{\prime}, k=1, \ldots, n$, and by $\rho^{\prime}$ the derivative of $b_{k}(\hat{c})$ and $\rho(\hat{c})$ respectively w.r.t. $\hat{c}$ at the point $\hat{c}=c$. Second, assuming also that $\rho \neq 0$, let us associate to $O$ the following rational function:

$$
A(z)=A_{O}(z)=\sum_{k=1}^{n} \frac{1}{\left(z-b_{k}\right)^{2}}+\frac{1}{\rho(1-\rho)} \sum_{k=1}^{n} \frac{\left(f^{n}\right)^{\prime \prime}\left(b_{k}\right)}{z-b_{k}}
$$

where $\left(f^{n}\right)^{\prime \prime}(z)$ is, of course, the second derivative of the $n$-iterate of $f$ w.r.t. $z$.

Comment 4 The numbers $\left(f^{n}\right)^{\prime \prime}\left(a_{k}\right)$ are connected directly to the uniformization of $f^{n}$ at the points $a_{k}$. For instance, if the orbit is hyperbolic (i.e. $|\rho| \neq 0,1$, then $\left(f^{n}\right)^{\prime \prime}\left(a_{k}\right) / \rho(\rho-1)=K^{\prime \prime}(0)$ where $K$ is the Konigs linearization function for the fixed point $a_{k}$ of $f^{n}$ normalizaed so that $K^{\prime}(0)=1$.

The above mentioned precise relation between the dynamical plane (function $A$ ) and the parameter plane (the derivative $\rho^{\prime}$ ) is described in

Theorem 2 Let $O$ be a periodic orbit of $f=f_{c}$ with multiplier $\rho \neq 1,0$, and $A=A_{O}$. Then:

(a) for $T=T_{c}$

$$
A(z)=(T A)(z)+\frac{\rho^{\prime}}{\rho} v_{*}(z)
$$

where

$$
v_{*}(z)=\frac{1}{z-c}=\left(T u_{*}\right)(z)
$$

with $u_{*}(z)=\ell z^{\ell-2}$.

$\left(a^{*}\right)$ (dual to $\left.(a)\right)$ for every backward invariant Beltrami form $\mu$ on a set $V$, if $\mu \cdot A$ is integrable on $V$, then

$$
\int_{V \backslash f^{-1}(V)} \mu(z) A(z) d \sigma_{z}=\frac{\rho^{\prime}}{\rho} \ell \int_{f^{-1}(V)} z^{\ell-2} \mu(z) d \sigma_{z} .
$$

Comment 5 In fact, Theorem 2 covers also the limit cases $\rho=0$ and $\rho=1$. Then one should consider (6) for $\hat{c}$ close to c, multiply both sides by $\rho(\hat{c})$ and $1-\rho(\hat{c})$ resp., and pass to a limit. Compare Section 2.2 and Section 6.

As an almost immediate corollary, we have 
Corollary 1.1 (Douady-Hubbard-Sullivan Theorem, see [4], [2] for $\ell=2$ )

Let $W$ by an $H$-component of $M_{\ell}$, and $\rho(c)$ be the multiplier of the attracting periodic orbit $P(c)=\left\{a_{1}(c), \ldots, a_{p}(c)\right\}$ of $f_{c}, c \in W$. Then $\rho: W \rightarrow \mathbf{D}$ is a $\ell-1$ covering map of $W$ onto the unit disc with a single branching point $c_{W} \in W$ where $\rho=0$.

See next Section for the proof.

By Corollary 1.1, any branch of the map $\rho^{\frac{1}{\ell-1}}$ extends to a conformal isomorphism of $W$ onto D.

Another corollary of Theorem 2 is the following

Theorem 3 Let $O$ be a periodic orbit of $f=f_{c}$ with multiplier $\rho$, such that $O$ is either attracting or parabolic (with $\rho \neq 1$ ). Denote by $Y$ the complex plane with the iterates $f^{n}(0), n>0$, and the orbit $O$ deleted. Then, for every $z \in Y$, the function

$$
\tilde{\mathbf{T}}(z):=\sum_{n=0}^{\infty} T^{n} \frac{1}{z-c}=\sum_{n>0}\left(T^{n} u_{*}\right)(z)
$$

is well defined, and

$$
\tilde{\mathbf{T}}(z)=\frac{\rho}{\rho^{\prime}} A(z) .
$$

Note that for the quadratic family $(\ell=2), \tilde{\mathbf{T}}(z)=2 \sum_{n>0} \sum_{f^{n}(w)=z} \frac{1}{\left(\left(f^{n}\right)^{\prime}(w)\right)^{2}}$.

See next Section for the proof.

For an application of Theorem 2 to parabolic periodic orbits, see Section 6 .

In fact, the inequality (1) for the H-components can be considered as a refinement of the Douady-Hubbard-Sullivan Theorem. Indeed, for any simply-connected bounded domain $\Omega$, the value $\operatorname{dist}(x, \partial \Omega$, for $x \in \Omega$, is closely related to the conformal radius $r(x)$ of $\Omega$ at $x$. The latter is defined as $r(x)=R_{x}^{\prime}(0)$, where, in turn, $R_{x}$ is the univalent map from the unit disc onto $\Omega$ normalized so that $R_{x}(0)=x$ and $R_{x}^{\prime}(0)>0$. By $1 / 4$-Koebe theorem,

$$
1 / 4 \leq \operatorname{dist}(x, \partial \Omega) / r(x) \leq 4 .
$$

That is, to prove Theorem 1, we need to estimate from above $r(x)$. On the other hand, for an H-component $W$, the conformal radius $r(c)$ is connected to the conformal isomorphism $\rho^{\frac{1}{\ell-1}}: W \rightarrow \mathbf{D}$ by the relation:

$$
r(c)=\frac{(\ell-1)|\rho|^{1-\frac{1}{\ell-1}}\left(1-|\rho|^{2}\right)}{\left|\rho^{\prime}\right|},
$$

where $\rho=\rho(c)$,

The inequality (1) for H-components will be an easy consequence from Propositions 2 and 3 on the behavior of the conformal radius, see Section 3. 


\section{$1.3 \quad$ Non-hyperbolic components}

It remains to consider $\mathrm{N}$-components of $M_{\ell}$. Then $c$ in such a component is characterized so that, there exists a subset $E$ of $J_{c}$ of a positive Lebesgue measure and a Beltrami form $\mu$ supported on $E$, which is backward (and forward) invariant, and $\|\mu\|_{L_{\infty}}=1$ [14], [16]. Moreover, $\mu$ is unique, in the sense, that for any other $\tilde{\mu}$ as above, there is a complex number $h$ of the modulus 1 , such that $\mu=h \tilde{\mu}$ a.e. (see Section 4)

Theorem 4 Let $W$ be an $N$-component of $M_{\ell}, c \in W, f=f_{c}$, and $\mu$ as above. Let $r$ be the conformal radius of $W$ at $c$. Then

$$
r=\left|\frac{1}{\pi} \int \frac{\mu(z)}{z-c} d \sigma_{z}\right|=\left|\frac{\ell}{\pi} \int z^{\ell-2} \mu(z) d \sigma_{z}\right| .
$$

See Section 4 for the proof. Clearly, (9) implies (1) for N-components.

\subsection{Acknowledgements.}

Most of this work has been done and written down during author's visits to IMPAN, Warsaw (September-November, 2001), IHES, Orsay (December, 2001), and Warwick Univ. (January-February, 2002). The author thanks A. Avila, A. Cheritat, A. Douady, H. Epstein, V. Marcovich, F. Przytycki, D. Ruelle, and J.-C. Yoccoz for stimulating and helpful discussions.

\section{Theorem 2 and its corollaries}

\subsection{The Ruelle transfer operator}

The statement and a hint. Remind that

$$
T g(z)=\sum_{w: f(w)=z} \frac{g(w)}{\left(f^{\prime}(w)\right)^{2}} .
$$

In view of Fact 1.1, we need to prove only Part $(a)$ of Theorem 2:

$$
A(z)=(T A)(z)+\frac{\rho^{\prime}}{\rho} \frac{1}{z-c}
$$

where:

$$
A(z)=\sum_{k=1}^{n} \frac{1}{\left(z-b_{k}\right)^{2}}+\frac{1}{\rho(1-\rho)} \sum_{k=1}^{n} \frac{\left(f^{n}\right)^{\prime \prime}\left(b_{k}\right)}{z-b_{k}}
$$


$O=\left\{b_{1}, \ldots, b_{n}\right\}$ is a periodic orbit of $f(z)=z^{\ell}+c$ of exact period $n$ and with the multiplier $\rho \neq 1,0$, and $\rho^{\prime}$ is the derivative of $\rho$ as a function of $c$.

There is a way to "guess" that something like the formula (10) should exist. Assume, for a moment, that the periodic orbit $O$ is attracting, more exactly, $0<|\rho|<1$, and consider the series (cf. [12])

$$
D_{\lambda}(z)=\sum_{k>0} \frac{\lambda^{k-1}}{\left(f^{k-1}\right)^{\prime}(c)\left(z-f^{k}(0)\right)} .
$$

It converges for $|\lambda|<|\rho|$ and for every $z$ in, say, the basin of infinity of $f$. On the other hand,

$$
\lambda\left(T D_{\lambda}\right)(z)=D_{\lambda}(z)-\frac{S(\lambda)}{z-c},
$$

where $S(\lambda)=\sum_{k>0} \lambda^{k-1} /\left(f^{k-1}\right)^{\prime}(c)$. It can be checked with help of the Konigs Linearization Theorem and the Cauchy formula, that, for fixed $z$, the function $D_{\lambda}(z)$ (as well as $S(\lambda)$ ) extends to a meromorphic function on the complex plane, with simple poles at the points $\lambda$, so that $\lambda^{n}=\rho^{j-1}$, for $j=1,2, \ldots$ If $R(z)$ and $R_{S}$ denote the residues of $D_{\lambda}(z)$ and $S(\lambda)$ resp. at one of those poles $\lambda_{0}$, then we come to the identity $\lambda_{0} T R(z)=R(z)-\frac{R_{S}}{z-c}$. In particular, take $\lambda_{0}=1$. Then calculations show that

$$
R(z)=\sum_{k=1}^{n} \frac{1}{\left(z-b_{k}\right)^{2}}-\sum_{k=1}^{n} \frac{E_{k}}{z-b_{k}}
$$

where

$$
E_{k}=\frac{\ell-1}{\left(f^{k-1}\right)^{\prime}\left(b_{1}\right)}\left(\frac{1}{\rho-1} \sum_{j=0}^{n-1} \frac{\left(f^{j}\right)^{\prime}\left(b_{1}\right)}{b_{1+j}}+\sum_{j=0}^{k-2} \frac{\left(f^{j}\right)^{\prime}\left(b_{1}\right)}{b_{1+j}}\right) .
$$

Key fact is that the residue $R_{S}$ of $S(\lambda)$ at the pole $\lambda_{0}=1$ turns out to be equal to $\rho^{\prime} / \rho$. Although we prove this fact below, the reasoning behind this is unclear.

Action of $T$ on Cauchy kernals The operator $T$ acts on functions $1 /(z-a)$, where $a$ is a parameter, as follows:

$$
T \frac{1}{z-a}=\frac{1}{f^{\prime}(a)}\left[\frac{1}{z-f(a)}-\frac{1}{z-c}\right]
$$

It can be checked easily, for example, calculating the integral $(1 / 2 \pi i) \int_{\Gamma} d w /\left(f^{\prime}(w)(f(w)-\right.$ $z)(w-a)$ with the contour $\Gamma$ enclosing $\infty$. Taking the derivative of (12) with respect to $a$, we get

$$
T \frac{1}{(z-a)^{2}}=\frac{1}{(z-f(a))^{2}}-\frac{\ell-1}{a f^{\prime}(a)}\left[\frac{1}{z-f(a)}-\frac{1}{z-c}\right]
$$


Let us show that

$$
\frac{1}{z-c}=\left(T u_{*}\right)(z)
$$

where $u_{*}(z)=\ell z^{\ell-2}$. Applying (12) and the Cauchy formula, we can write:

$$
\left(T u_{*}\right)(z)=\frac{1}{2 \pi i} \int_{\Gamma} u_{*}(a)\left(T \frac{1}{a-z}\right) d a=\frac{1}{2 \pi i} \int_{\Gamma} \frac{1}{a}\left[\frac{1}{z-c}-\frac{1}{z-f(a)}\right] d a=\frac{1}{z-a} .
$$

Proof of (10). First, let's check that $A(z)=R(z)$, i.e. we need to show that $\frac{\left(f^{n}\right)^{\prime \prime}\left(b_{k}\right)}{\rho(\rho-1)}=E_{k}$. One writes:

$$
\begin{gathered}
(\rho-1) E_{k}=\frac{\ell-1}{\left(f^{k-1}\right)^{\prime}\left(b_{1}\right)}\left[\sum_{j=0}^{n-1} \frac{\left(f^{j}\right)^{\prime}\left(b_{1}\right)}{b_{1+j}}+\rho \sum_{j=0}^{k-2} \frac{\left(f^{j}\right)^{\prime}\left(b_{1}\right)}{b_{1+j}}-\sum_{j=0}^{k-2} \frac{\left(f^{j}\right)^{\prime}\left(b_{1}\right)}{b_{1+j}}\right]= \\
\frac{\ell-1}{\left(f^{k-1}\right)^{\prime}\left(b_{1}\right)}\left[\sum_{j=k-1}^{n-1} \frac{\left(f^{j}\right)^{\prime}\left(b_{1}\right)}{b_{1+j}}+\rho \sum_{j=0}^{k-2} \frac{\left(f^{j}\right)^{\prime}\left(b_{1}\right)}{b_{1+j}}\right]=(\ell-1) \sum_{j=0}^{n-1} \frac{\left(f^{j}\right)^{\prime}\left(b_{k}\right)}{b_{j+k}}=\frac{\left(f^{n}\right)^{\prime \prime}\left(b_{k}\right)}{\left(f^{n}\right)^{\prime}\left(b_{k}\right)}=\frac{\left(f^{n}\right)^{\prime \prime}\left(b_{k}\right)}{\rho} .
\end{gathered}
$$

We use here notation $b_{n+j}=b_{j}$ and the identities $\rho /\left(f^{k-1}\right)^{\prime}\left(b_{1}\right)=\left(f^{n-k+1}\right)^{\prime}\left(b_{k}\right)$ and $\left(f^{n-k+1}\right)^{\prime}\left(b_{k}\right)\left(f^{j}\right)^{\prime}\left(b_{1}\right)=\left(f^{n-k+1+j}\right)^{\prime}\left(b_{k}\right)$. Now, using (12) and (13), we see that

$$
T R(z)=R(z)+\frac{F}{z-c}
$$

where

$$
F=\sum_{k=1}^{n}\left[\frac{E_{k}}{f^{\prime}\left(b_{k}\right)}+\frac{\ell-1}{b_{k} f^{\prime}\left(b_{k}\right)}\right] .
$$

It remains to show that $F=-\rho^{\prime} / \rho$. Make the following transformations:

$$
\begin{gathered}
F=\sum_{k=1}^{n}\left[\frac{E_{k}}{f^{\prime}\left(b_{k}\right)}+\frac{\ell-1}{b_{k} f^{\prime}\left(b_{k}\right)}\right]=\sum_{k=1}^{n}\left[\frac{\ell-1}{\left(f^{k}\right)^{\prime}\left(b_{1}\right)}\left(\frac{1}{\rho-1} \sum_{j=0}^{n-1} \frac{\left(f^{j}\right)^{\prime}\left(b_{1}\right)}{b_{1+j}}+\sum_{j=0}^{k-2} \frac{\left(f^{j}\right)^{\prime}\left(b_{1}\right)}{b_{1+j}}\right)+\frac{\ell-1}{b_{k} f^{\prime}\left(b_{k}\right)}\right] \\
=\sum_{k=1}^{n} \frac{\ell-1}{\left(f^{k}\right)^{\prime}\left(b_{1}\right)}\left(\frac{1}{\rho-1} \sum_{j=0}^{n-1} \frac{\left(f^{j}\right)^{\prime}\left(b_{1}\right)}{b_{1+j}}+\sum_{j=0}^{k-1} \frac{\left(f^{j}\right)^{\prime}\left(b_{1}\right)}{b_{1+j}}\right)= \\
(\ell-1) \sum_{j=0}^{n-1} \frac{\left(f^{j}\right)^{\prime}\left(b_{1}\right)}{b_{1+j}}\left(\frac{1}{\rho-1} \sum_{k=1}^{n} \frac{1}{\left(f^{k}\right)^{\prime}\left(b_{1}\right)}+\sum_{k=j+1}^{n} \frac{1}{\left(f^{k}\right)^{\prime}\left(b_{1}\right)}\right)= \\
(\ell-1) \sum_{j=0}^{n-1} \frac{\left(f^{j}\right)^{\prime}\left(b_{1}\right)}{b_{1+j}}\left(\frac{\rho}{\rho-1} \sum_{k=1}^{n} \frac{1}{\left(f^{k}\right)^{\prime}\left(b_{1}\right)}-\sum_{k=1}^{j} \frac{1}{\left(f^{k}\right)^{\prime}\left(b_{1}\right)}\right) .
\end{gathered}
$$


Now we use identities

$$
\frac{\partial f^{m}}{\partial c}(z)=\left(f^{m}\right)^{\prime}(z) \sum_{j=1}^{m} \frac{1}{\left(f^{j}\right)^{\prime}(z)}
$$

so that the derivative of $b_{1}$ w.r.t. $c$,

$$
b_{1}^{\prime}=\frac{\frac{\partial f^{n}}{\partial c}\left(b_{1}\right)}{1-\rho}=\frac{\rho}{1-\rho} \sum_{k=1}^{n} \frac{1}{\left(f^{k}\right)^{\prime}\left(b_{1}\right)},
$$

and continue:

$$
\begin{gathered}
F=-(\ell-1) \sum_{j=0}^{n-1} \frac{\left(f^{j}\right)^{\prime}\left(b_{1}\right)}{b_{1+j}}\left(b_{1}^{\prime}+\sum_{k=1}^{j} \frac{1}{\left(f^{k}\right)^{\prime}\left(b_{1}\right)}\right)=-(\ell-1) \sum_{j=0}^{n-1} \frac{1}{b_{1+j}}\left(\left(f^{j}\right)^{\prime}\left(b_{1}\right) b_{1}^{\prime}+\frac{\partial f^{j}}{\partial c}\left(b_{1}\right)\right) \\
=-(\ell-1) \sum_{j=0}^{n-1} \frac{b_{j+1}^{\prime}}{b_{j+1}}=-\frac{\rho^{\prime}}{\rho} .
\end{gathered}
$$

\subsection{Douady-Hubbard-Sullivan Theorem}

Let $W$ by an H-component of $M_{\ell}$, and $\rho(c)$ be the multiplier of the attracting periodic orbit $P(c)=\left\{a_{1}(c), \ldots, a_{p}(c)\right\}$ of $f_{c}, c \in W$. We prove that $\rho: W \rightarrow \mathbf{D}$ is a $\ell-1$-covering map of $W$ onto the unit disc with a single branching point $c_{W} \in W$ where $\rho\left(c_{W}\right)=0$.

A priori, the map $\rho: W \rightarrow \mathbf{D}$ is a branching covering, because its limit values are of the modulus 1 . Hence, it is enough to prove two facts:

(1) $\rho^{\prime} \neq 0$ for any $c \in W$ where $\rho \neq 0$,

(2) if $\rho(\tau)=0$ for some $c=\tau \in W$, then necessarily $\rho^{\prime}(\tau)=\rho^{\prime \prime}(\tau)=\ldots=$ $\rho^{(\ell-2)}(\tau)=0$ while $\rho^{(\ell-1)}(\tau) \neq 0$.

To prove (1), assume $\rho^{\prime}=0$ for some $c \in W$. Then we get, for $f=f_{c}$ and $P=P(c)$, according to Theorem $2\left(a^{*}\right)$, that $\int_{C} \mu A d \sigma=0$, where $A=A_{P}$, and $C$ is a fundamental annulus in the basin of attarction of the periodic orbit $P$ of $f$ around, say, the point $a_{1}$ of $P$, and for any $\mu \in L_{\infty}(C)$. Observe that $A$ is intagrable on $V$ because $V$ is disjoint with a neighborhood of the set $P$. Now, choosing $\mu$ so that $\mu A=|A|$ on $C$, we get that $A=0$ on $C$, a contradiction with the fact that $A$ is a non-zero rational function.

Let's prove (2). Let $\rho(\tau)=0$, for some $c=\tau \in W$. Denote by $a_{p}$ the point of $P(\tau)$ such that $a_{p}=0$, and $c_{p}=f_{\tau}^{p}(0)$. Denote also by $a_{p}^{\prime}$ and $c_{p}^{\prime}$ the derivatives of $a_{p}, c_{p}$ w.r.t. $c$ at $\tau$. Differentiating the equality $\rho(c)=\ell^{p}\left(a_{1}(c) \ldots a_{p}(c)\right)^{\ell-1} \ell-1$ times at the point $c=\tau$, we have:

$$
\rho^{\prime}=\ldots=\rho^{(\ell-2)}=0
$$

while $\rho^{(\ell-1)}=\ell !\left(f^{p-1}\right)^{\prime}(\tau)\left(a_{p}^{\prime}\right)^{\ell-1}$. (Here, as always, $\left(f^{k}\right)^{\prime}(x)=f^{\prime}(x) \ldots f^{\prime}\left(f^{k-1}(x)\right)$ is the derivative w.r.t. $z$.) Thus it is enough to prove 
Proposition 1 At $c=\tau$,

$$
c_{p}^{\prime}=a_{p}^{\prime} \neq 0
$$

Proof. (cf. [11], [20]). We have at $c=\tau$ :

$$
\begin{gathered}
a_{p}^{\prime}=\frac{\left(\partial f^{p} / \partial c\right)\left(a_{p}\right)}{1-\rho}=\frac{\partial f^{p}}{\partial c}\left(a_{p}\right)=\frac{\partial f^{p}}{\partial c}(0)=c_{p}^{\prime} \\
=\left(f^{p-1}\right)^{\prime}(f(0)) S_{p}
\end{gathered}
$$

where

$$
S_{p}=\sum_{k=0}^{p-1} \frac{1}{\left(f^{k}\right)^{\prime}(f(0))} .
$$

Introduce

$$
\psi(z)=\sum_{k=0}^{p-1} \frac{1}{\left(f^{k}\right)^{\prime}(f(0))} \frac{1}{z-f^{k+1}(0)} .
$$

Applying (12), we see that

$$
T \psi(z)=\psi(z)-\frac{S_{p}}{z-\tau}
$$

Assuming $S_{p}=0$ and then repeating the argument in the proof that $\rho^{\prime} \neq 0$ if $\rho \neq 0$, we get a contradiction. Thus $S_{p} \neq 0$, and, therefore,

$$
a_{p}^{\prime}=c_{p}^{\prime}=\left(f^{p-1}\right)^{\prime}(f(0)) S_{p} \neq 0 .
$$

\subsection{The function $\sum_{n=0}^{\infty} T^{n} \frac{1}{z-c}$.}

Denote

$$
\tilde{\mathbf{T}}(z)=\sum_{n=0}^{\infty} T^{n} \frac{1}{z-c}=\sum_{n=0}^{\infty} \sum_{f^{n}(w)=z} \frac{1}{\left(\left(f^{n}\right)^{\prime}(w)\right)^{2}(w-c)}
$$

According to (14),

$$
\tilde{\mathbf{T}}(z)=\sum_{n>0}\left(T^{n} u_{*}\right)(z)=\ell \sum_{n>0} \sum_{f^{n}(w)=z} \frac{w^{\ell-2}}{\left(\left(f^{n}\right)^{\prime}(w)\right)^{2}} .
$$

If $z \in Y$, i.e. $z$ is not in the closure of the iterates of the critical point, then it is easy to see that $\tilde{\mathbf{T}}(z)$ is well defined and holomorphic in a neighborhood of $z$; this follows from the Koebe Distortion Theorem applied to a neighborhood $C$ of $z$ combined 
with the estimate $\int_{C} \sum_{n>0}\left|\left(T^{n} u_{*}\right)(z)\right| d \sigma_{z} \leq L \sum_{n>0}$ area $\left(f^{-n}(C)\right)<\infty$, where the constant $L=\ell\left[\operatorname{diam}\left(K_{c}\right)\right]^{\ell-2}$. Now we have from Theorem 2,

$$
\sum_{k=0}^{n-1} T^{k} \frac{1}{z-c}=\frac{\rho}{\rho^{\prime}}\left(A(z)-\left(T^{n} A\right)(z)\right)
$$

Since $Y$ is open and connected and $\tilde{\mathbf{T}}$ is holomorphic in $Y$, by analytic continuation, it is enough to prove the statement for $z$ in the basin of of infinity of $f$. For each such $z$, find a neighborhood $C$ such that $f^{-n}(C)$ are pairwise disjoint. If $O$ is attracting, then the set $V:=\cup_{n>0} f^{-n}(C)$ stays away from a neighborhood of $O$, hence, $A$ is integrable on $V$ in this case. If $O$ is parabolic, this is true as well. Indeed, then functions $1 /|z-a|^{2}$, for $a \in O$, are integrable in the intersection of a neighborhood of the point $a$ with the basin of infinity, as it follows from the local dynamics at parabolic points. Thus, in any case, one can write $\sum_{n>0} \int_{C}\left|T^{n} A\right| d \sigma \leq$ $\int_{\cup_{n>0} f^{-n}(C)}|A| d \sigma<\infty$, in particular, $\int_{C}\left|\left(T^{n} A\right)(z)\right| d \sigma_{z} \rightarrow 0$ as $n \rightarrow 0$. Since $\left(T^{n} A\right)(z)$ are holomorphic in $C,\left(T^{n} A\right)(z) \rightarrow 0$ uniformly on compacts in $C$, and Theorem 3 follows from (15).

Comment 6 Considering limits of corresponding functions when the parameter tends to the boundary of $H$-component from inside, one can generalize the above statement for Siegel as well as Cremer periodic orbit.

\section{Proof of Theorem 1 for H-components}

For the hyperbolic components of $M_{\ell}$, Theorem 1 will be a consequence of Propositions 2 and 3 below, where we will apply Proposition 2 for $|\rho|$ close to 1 and Proposition 3 for $|\rho|$ close to zero. Let $W$ be a H-component of $M_{\ell}, c \in W, f=f_{c}$, $P=\left\{a_{1}, \ldots, a_{p}\right\}$ be the attracting periodic orbit of $f$ with multiplier $\rho$, and $A(z)$ be the function associated with the orbit $P$.

\subsection{A special Beltrami form: Proposition 2}

We assume here that $\rho \neq 0$. Pick up the point $a_{1}$. Then let $\Phi$ be the Konigs linearization function, which is defined and univalent in a neighborhood $U$ of $a_{1}$, normalized so that $\Phi\left(a_{1}\right)=0$ and $\Phi^{\prime}\left(a_{1}\right)=1$, and

$$
\Phi \circ f^{p}(z)=\rho \Phi(z)
$$

for $z \in U$. One can assume that $\Phi(U)$ is a round disc centered at 0 . Let $K=\Phi^{-1}$. Fix real $m>0$ small enough, and define a fundamental annulus

$$
C:=K(\{|\rho| m<|w|<m\}) .
$$


Now set, for every $z \in C$,

$$
\mu(z):=\left\{\frac{\Phi(z)}{|\Phi(z)|} \frac{\left|\Phi^{\prime}(z)\right|}{\Phi^{\prime}(z)}\right\}^{2} .
$$

Then spread $\mu_{C}$ out to the set $V=\cup_{n \geq 0} f^{-n}(C)$ according to the rule (backward invariance): if $f^{n}(z) \in C$, for some $n>0$, then $\mu(z)=\mu\left(f^{n}(z)\right) \frac{\left|\left(f^{n}\right)^{\prime}(z)\right|^{2}}{\left(\left(f^{n}\right)^{\prime}(z)\right)^{2}}$.

Comment 7 It is easy to check that $\mu$ defined near $a_{1}$ as above is authomatically a forward invariant form as well. It allows us to consider $\mu$ as a function on the whole basin of attraction $F$ of $f$.

\section{Proposition 2}

$$
2 \rho \log \frac{1}{|\rho|}=\ell \frac{\rho^{\prime}}{\pi} \int_{f^{-1}(V)} z^{\ell-2} \mu(z) d \sigma_{z}=\ell \frac{\rho^{\prime}}{\pi} \int_{F} z^{\ell-2} \mu(z) d \sigma_{z},
$$

where $f^{-1}(V)=\cup_{n>0} f^{-n}(C)$.

Proof. In polar coordinates, $w=\tau \exp (i \theta)$ and then

$$
\begin{gathered}
\int_{C} \mu_{C}(z) A(z) d \sigma_{z}=\int_{\{|\rho| m<|w|<m\}} \mu_{C}(K(w)) A(K(w))\left|K^{\prime}(w)\right|^{2} d \sigma_{w}= \\
\int_{\{|\rho| m<|w|<m\}} \frac{w^{2}}{|w|^{2}}\left(K^{\prime}(w)\right)^{2} A(K(w)) d \sigma_{w}=2 \pi \int_{|\rho| m}^{m} \frac{d \tau}{\tau} \frac{1}{2 \pi i} \int_{|w|=\tau} w\left(K^{\prime}(w)\right)^{2} A(K(w)) d w \\
=2 \pi \log \frac{1}{|\rho|},
\end{gathered}
$$

where we use that $w\left(K^{\prime}(w)\right)^{2} A(K(w)) \sim \frac{1}{w}$ as $w \rightarrow 0$. It remains to apply Theorem $2\left(a^{*}\right)$.

\subsection{Proposition 3}

Blaschke products. Pick up any $c \in W, f=f_{c}, P=P(c), \rho=\rho(c)$. Let $\Omega$ be a component of the immediate basin of attraction of the attracting periodic orbit $P$ of $f$, which, for definicity, contains 0 and the point $a$ of $P$. Let $\phi: \mathbf{D} \rightarrow \Omega$ be a Riemann map, normalized so that $\phi(0)=a$. Then the conjugate $\operatorname{map} B(w):=\phi^{-1} \circ f^{p} \circ \phi(w)$ is a Blaschke product, such that $B(0)=0, \rho=B^{\prime}(0)$, and $B$ has a single critical point of order $\ell$. Then it is easy to see that $\phi$ can be chosen so that

$$
B(w)=B_{a, \ell}(w)=K_{1}\left(\left[K_{0}(w)\right]^{\ell}\right)
$$


where

$$
K_{0}(w)=\frac{w+a}{1+\bar{a} w}, K_{1}(w)=\frac{w-b}{1-\bar{b} w}
$$

and $b=a^{\ell}$, for some $0<|a|<1$. Hence,

$$
B^{\prime}(w)=K_{1}^{\prime}\left(\left[K_{0}(w)\right]^{\ell}\right) \ell\left[K_{0}(w)\right]^{\ell-1} K_{0}^{\prime}(w),
$$

so that

$$
\rho=\frac{\ell a^{\ell-1}\left(1-|a|^{2}\right)}{1-|a|^{2 \ell}} .
$$

Therefore, given $\rho, 0 \leq|\rho|<1$, the number $|a|$ is determined as the unique solution of the equation

$$
|\rho|=\frac{\ell|a|^{\ell-1}\left(1-|a|^{2}\right)}{1-|a|^{2 \ell}}
$$

such that $0 \leq|a|<1$, and then $a$ is unique determined as well from (17), up to a rotation by $1 /(\ell-1)$ root of unity.

By continuity, there exists $a_{*}>0,0<a_{*}<0.5$, be so that:

for every $a$ with $|a| \leq a_{*}$ and every $w$ with $|w| \leq \frac{1}{2}$, we have:

$$
\left|\frac{w+a}{1+\bar{a} w}\right|^{2} \leq \frac{1}{3}-\frac{1}{24}
$$

for every $b$ with $|b| \leq a_{*}^{2}$ and every $w$ with $|w| \leq \frac{1}{3}-\frac{1}{24}$, we have:

$$
\left|\frac{w-b}{1-\bar{b} w}\right| \leq \frac{1}{3}
$$

Then we have

Fact 3.1 For every $a,|a| \leq a_{*}$, and every $\ell \geq 2$,

$$
\left\{\frac{1}{3}<|w|<\frac{1}{2}\right\} \subset B_{a, \ell}^{-1}\left(\mathbf{D}_{1 / 3}\right) \backslash \mathbf{D}_{1 / 3},
$$

where $\mathbf{D}_{r}=\{|w|<r\}$.

Uniformization of the function $A$ We carry out some calculations, which are collected in

Lemma 3.1 Let $0<|\rho|<1$ and $B(w)=B_{a, \ell}(w)=\phi^{-1} \circ f^{p} \circ \phi(w)$. Then:

$$
\left(\phi^{\prime}(w)\right)^{2} A(\phi(w))=\frac{1}{w^{2}}-\frac{B^{\prime \prime}(0)}{\rho(\rho-1)} \frac{1}{w}+l(w),
$$

where $l$ is a function, which is holomorphic in the unit disc $\mathbf{D}$. 


$$
B^{\prime \prime}(0)=\frac{\ell a^{\ell-2}\left(1-|a|^{2}\right)}{\left(1-|a|^{2 \ell}\right)^{2}} \cdot\left\{(\ell-1)\left(1-|a|^{2 \ell+2}\right)-(\ell+1)\left|a^{2}\right|\left(1-|a|^{2 \ell-2}\right)\right\}
$$

Proof. ¿From the definition of $A$,

$$
\left(\phi^{\prime}(w)\right)^{2} A(\phi(w))=\frac{\left(\phi^{\prime}(w)\right)^{2}}{(\phi(w)-\phi(0))^{2}}-\frac{1}{\rho(\rho-1)} \frac{\left.\left(f^{p}\right)^{\prime \prime}(\phi(w))\left(\phi^{\prime}(w)\right)^{2}\right)}{\phi(w)-\phi(0)}+l_{1}(w),
$$

where $l_{1}$ is a function holomorphic in $\mathbf{D}$. After differentiating twice the identity $f^{p} \circ \phi(w)=\phi \circ B(w)$ at $w=0$, we get:

$$
\left(f^{p}\right)^{\prime \prime}(\phi(0))\left(\phi^{\prime}(0)\right)^{2}=\phi^{\prime \prime}(0) \rho(\rho-1)+\phi^{\prime}(0) B^{\prime \prime}(0) .
$$

Then

$$
\begin{gathered}
\frac{\left(\phi^{\prime}(w)\right)^{2}}{(\phi(w)-\phi(0))^{2}}-\frac{1}{\rho(\rho-1)} \frac{\left(f^{p}\right)^{\prime \prime}(\phi(w))\left(\phi^{\prime}(w)\right)^{2}}{\phi(w)-\phi(0)} \\
=\frac{1}{w^{2}}\left(\frac{1+w \phi^{\prime \prime}(0) / \phi^{\prime}(0)+O\left(|w|^{2}\right)}{1+w \cdot \phi^{\prime \prime}(0) / 2 \phi^{\prime}(0)+O\left(|w|^{2}\right)}\right)^{2}-\frac{1}{w \phi^{\prime}(0)}\left(\phi^{\prime \prime}(0)+\phi^{\prime}(0) \cdot \frac{B^{\prime \prime}(0)}{\rho(\rho-1)}\right)(1+O|w|) \\
=\frac{1}{w^{2}}\left(1+w \frac{\phi^{\prime \prime}(0)}{\phi^{\prime}(0)}+O\left(|w|^{2}\right)\right)-\frac{1}{w}\left(\frac{\phi^{\prime \prime}(0)}{\phi^{\prime}(0)}+\frac{B^{\prime \prime}(0)}{\rho(\rho-1)}+O(|w|)\right)+O(1) \\
=\frac{1}{w^{2}}-\frac{1}{w} \frac{B^{\prime \prime}(0)}{\rho(\rho-1)}+O(1) .
\end{gathered}
$$

Let us prove the second part. We have:

$$
\begin{gathered}
B^{\prime}(w)=K_{1}^{\prime}\left(\left[K_{0}(w)\right]^{\ell}\right) \ell\left[K_{0}(w)\right]^{\ell-1} K_{0}^{\prime}(w), \\
B^{\prime \prime}(0)=K_{1}^{\prime \prime}(b)\left[\ell a^{\ell-1}\right]^{2}\left[K_{0}^{\prime}(0)\right]^{2}+K_{1}^{\prime}(b) \ell(\ell-1) a^{\ell-2}\left[K_{0}^{\prime}(0)\right]^{2}+K_{1}^{\prime}(b) \ell a^{\ell-1} K_{0}^{\prime \prime}(0) . \\
\rho=K_{1}^{\prime}(b) \ell a^{\ell-1} K_{0}^{\prime}(0)=\frac{\ell a^{\ell-1}\left(1-|a|^{2}\right)}{1-|a|^{2 \ell}}
\end{gathered}
$$

and finally,

$$
\begin{aligned}
B^{\prime \prime}(0) & =\frac{\ell a^{\ell-2}\left(1-|a|^{2}\right)}{\left(1-|a|^{2 \ell}\right)^{2}} \cdot\left\{2 \ell|a|^{2 \ell}\left(1-|a|^{2}\right)+(\ell-1)\left(1-|a|^{2}\right)\left(1-|a|^{2 \ell}\right)-2|a|^{2}\left(1-|a|^{2 \ell}\right)\right\} \\
& =\frac{\ell a^{\ell-2}\left(1-|a|^{2}\right)}{\left(1-|a|^{2 \ell}\right)^{2}} \cdot\left\{(\ell-1)\left(1-|a|^{2 \ell+2}\right)-(\ell+1)\left|a^{2}\right|\left(1-|a|^{2 \ell-2}\right)\right\} .
\end{aligned}
$$




\section{The statement.}

Proposition 3 If $\rho$ is such that

$$
|\rho| \leq \frac{\ell a_{*}^{\ell-1}\left(1-a_{*}^{2}\right)}{1-a_{*}^{2 \ell}}
$$

then

$$
2 \pi\left(\frac{1}{2}-\frac{1}{3}\right)\left|\frac{B^{\prime \prime}(0)}{1-\rho}\right| \leq\left|\rho^{\prime}\right| \cdot \ell \cdot \int_{F_{c}}|z|^{\ell-2} d \sigma_{z} .
$$

Proof. For every $\rho$ as above, we find $B=B_{a, \ell}$, where $|a| \leq a_{*}$. According to Fact 3.1, we can define a fundamental annulus $C:=\phi^{-1}\left(C_{B}\right)$, where $C_{B}:=$ $B^{-1}\left(\mathbf{D}_{1 / 3}\right) \backslash \mathbf{D}_{1 / 3}$ contains the round annulus $C_{1 / 3,1 / 2}=\left\{\frac{1}{3}<|w|<\frac{1}{2}\right\}$. Define $\mu(z)$ for $z=\phi(w)$, where $w \in C_{B}$, to be equal:

- $\mu(\phi(w))=0$ if $w \in C_{B} \backslash C_{1 / 3,1 / 2}$,

$$
\mu(\phi(w))=\frac{\left(\phi^{\prime}(w)\right)^{2}}{\left|\phi^{\prime}(w)\right|^{2}} \frac{w}{|w|}
$$

if $w \in C_{1 / 3,1 / 2}$.

Then we have, in polar coordinate $w=\tau \exp (i \theta)$ :

$$
\begin{aligned}
& \int_{C} \mu(z) A(z) d \sigma_{z}=\int_{\{1 / 3<|w|<1 / 2\}} \mu(\phi(w)) A(\phi(w))\left|\phi^{\prime}(w)\right|^{2} d \sigma_{w}= \\
& 2 \pi \int_{1 / 3}^{1 / 2} d \tau \frac{1}{2 \pi i} \int_{|w|=\tau}\left(\phi^{\prime}(w)\right)^{2} A(\phi(w)) d w=2 \pi\left(\frac{1}{2}-\frac{1}{3}\right) \frac{B^{\prime \prime}(0)}{\rho(1-\rho)},
\end{aligned}
$$

where Lemma 3.1 is used. It remains to apply Theorem $2\left(a^{*}\right)$.

Proof of Theorem 1 for H-components. It is enough to estimate the conformal radius $r$ at the point $c$ of a hyperbolic component $W$ of $M_{\ell}$. As we know,

$$
r=\frac{(\ell-1)|\rho|^{1-\frac{1}{\ell-1}}\left(1-|\rho|^{2}\right)}{\left|\rho^{\prime}\right|} .
$$

Given $\ell \geq 2$, consider two cases:

(1)

$$
|\rho|>\frac{\ell a_{*}^{\ell-1}\left(1-a_{*}^{2}\right)}{1-a_{*}^{2 \ell}} .
$$


Denote $t=|\rho|^{1 /(\ell-1)}$, then

$$
t \geq a_{*}\left[\frac{\ell}{1+a^{2}+\ldots+a_{*}^{2 \ell-2}}\right]^{1 /(\ell-1)}>a_{*},
$$

and applying Proposition 2,

$$
\begin{gathered}
r=\frac{(\ell-1)|\rho|^{1-\frac{1}{\ell-1}}\left(1-|\rho|^{2}\right)}{|\rho| \log \frac{1}{\rho \mid}} \cdot \frac{|\rho| \log \frac{1}{|\rho|}}{\left|\rho^{\prime}\right|} \leq \\
\frac{(\ell-1)|\rho|^{1-\frac{1}{\ell-1}}\left(1-|\rho|^{2}\right)}{|\rho| \log \frac{1}{\rho \mid}} \cdot \frac{\ell}{2 \pi} \int_{F_{c}}\left|z^{\ell-2}\right| d \sigma_{z} \leq \frac{1-t^{\ell-1}}{t \log \frac{1}{t}} \cdot \frac{\ell}{\pi} \int_{F_{c}}\left|z^{\ell-2}\right| d \sigma_{z} \leq(\ell-1) \mathbf{L}_{1} \ell \int_{F_{c}}\left|z^{\ell-2}\right| d \sigma_{z},
\end{gathered}
$$

where $\mathbf{L}_{1}=\sup \left\{(1-t) / t \log (1 / t): a_{*} \leq t<1\right\}$ is an absolute constant.

(2)

$$
|\rho| \leq \frac{\ell a_{*}^{\ell-1}\left(1-a_{*}^{2}\right)}{1-a_{*}^{2 \ell}} .
$$

Then $\rho=\frac{\ell a^{\ell-1}\left(1-|a|^{2}\right)}{1-|a|^{2 \ell}}$, where $|a| \leq a_{*}$. To estimate $r$, we now apply Proposition 3 and Lemma 3.1:

$$
\begin{aligned}
& r=\frac{(\ell-1)|\rho|^{1-\frac{1}{\ell-1}}\left(1-|\rho|^{2}\right)}{\left|\rho^{\prime}\right|} \leq \frac{(\ell-1)|\rho|^{1-\frac{1}{\ell-1}}\left(1-|\rho|^{2}\right)}{\left|B^{\prime \prime}(0)\right|} \cdot \frac{3|1-\rho|}{\pi} \cdot \ell \int_{F_{c}}\left|z^{\ell-2}\right| d \sigma_{z} \leq \\
& \frac{(\ell-1)\left(\frac{\ell|a|^{\ell-1}\left(1-|a|^{2}\right)}{1-|a|^{2 \ell}}\right)^{1-\frac{1}{\ell-1}}}{\left|\frac{\ell a^{\ell-2}\left(1-|a|^{2}\right)}{\left(1-|a|^{2 \ell}\right)^{2}} \cdot\left\{(\ell-1)\left(1-|a|^{2 \ell+2}\right)-(\ell+1)\left|a^{2}\right|\left(1-|a|^{2 \ell-2}\right)\right\}\right|} \cdot \frac{6}{\pi} \cdot \ell \int_{F_{c}}\left|z^{\ell-2}\right| d \sigma_{z} \leq \\
& \left(\frac{1+|a|^{2}+\ldots+|a|^{2 \ell-2}}{\ell}\right)^{\frac{1}{\ell-1}} \cdot \frac{1}{\left(1-\frac{\ell+1}{\ell-1}|a|^{2}\right)+|a|^{2 \ell}\left(\frac{\ell+1}{\ell-1}-|a|^{2}\right)} \cdot \frac{6}{\pi} \cdot \ell \int_{F_{c}}\left|z^{\ell-2}\right| d \sigma_{z} \leq \\
& \frac{12}{\pi} \cdot \ell \int_{F_{c}}\left|z^{\ell-2}\right| d \sigma_{z} .
\end{aligned}
$$

End of the proof of Theorem 1 for the H-components.

\section{Non-hyperbolic components}

Here we prove Theorem 5 thus completing the proof of Theorem 1 . Let $W$ be an N-component of $M_{\ell}$, and $c=\tau \in W$. Let $R_{\tau}$ be the univalent map from the unit disc $\mathbf{D}$ onto $W$ normalized so that $R_{\tau}(0)=\tau$. In the first step, we construct the Riemann map $R_{\tau}: t \in \mathbf{D} \mapsto c(t) \in W$. In the second step we calculate the conformal radius $r=\left|R_{\tau}^{\prime}(0)\right|$ of $W$ at $\tau$. 


\subsection{The Riemann map}

The construction we describe in this subsection is known, at least, implicitely, see e.g. [16]. As it follows from the $\lambda$-lemma [14], for any $c_{0} \in W, c_{0} \neq \tau$, the maps $f_{c_{0}}$ and $f_{\tau}$ are conjugate by a quasi-conformal homeomorphism $h$ of the plane:

$$
h \circ f_{\tau}=f_{c_{0}} \circ h,
$$

$h$ is conformal off $J_{\tau}$, and $h(z) \sim z$ at infinity. Let $\mu=\mu_{h}$ be the Beltrami coefficient of $h: \mu=(\partial h / \partial \bar{z}) /(\partial h / \partial z)$. Then:

(1) $\mu=0$ outside of $J_{\tau}, 0<\|\mu\|_{L_{\infty}}<1$, and, from the conjugacy equation,

(2)

$$
\mu\left(f_{\tau}(z)\right)=\mu(z)\left(\frac{f_{\tau}^{\prime}(z)}{\left|f_{\tau}^{\prime}(z)\right|}\right)^{2}
$$

a.e.

First, we prove the uniqueness:

Proposition 4 If $\nu$ satisfies (1)-(2), then there exists a number $\psi \neq 0$ such that $\nu=\psi \mu$ a.e.

Proof. For any $\nu$ which satsifies (1)-(2), define $\hat{\nu}(z)=\int \nu(w) d \sigma_{w} /(z-w)$. Then (see[11]) $\hat{\nu}$ is a continuous function on the plane, $\hat{\nu}(\tau) \neq 0$, and $Q(z):=\hat{\nu}(z) / \hat{\nu}(\tau)$ satisfies the functional equation $f_{\tau}^{\prime}(z) Q(z)-Q\left(f_{\tau}(z)\right)=-1$. Iterating this equation for $z$ in the basin of infinity of $f_{\tau}$, we obtain that $Q(z)=-\sum_{n>0} 1 /\left(f_{\tau}^{n}\right)^{\prime}(\tau)$ i.e. is defined uniquely. Therefore, $\hat{\nu}(z)=\psi \hat{\mu}(z)$ with $\psi:=\hat{\nu}(\tau) / \hat{\mu}(\tau)$. Now, define $k(z):=\nu(z)-\psi \hat{\mu}(z)$. Then, for every $z, \int k(w) d \sigma_{w} /(z-w)=\hat{\nu}(z)-\psi \hat{\mu}(z)=0$, which implies that $k=0$ a.e.

¿From now on, let's fix any $\mu$ which satisfies (1)-(2) and normalized so that $\|\mu\|_{L_{\infty}}=1$. For every $t,|t|<1$, denote by $\mu_{t}$ the Beltrami coefficient, which is equal to $t \mu(z)$ for $z \in E$ and 0 otherwise. Since ||$\mu_{t}||_{L_{\infty}}=|t|<1$ and $\mu_{t}$ is zero off $J_{\tau}$, there exists and unique so-called normal solution of the Beltrami equation (see [1], Ch. V, Th. 1), i.e. a quasi-conformal homeomorphism $h^{t}$ of the plane with the Beltrami coefficient $\mu_{t}$ and such that $h^{t}(0)=0, h^{t}(z) / z \rightarrow 1$ as $z \rightarrow \infty$. Obviously, $h^{0}$ is the identity. Since $\mu_{t}$ satisfies the invariance condition (2), and by the normalizations,

$$
h^{t} \circ f_{\tau} \circ\left(h^{t}\right)^{-1}(z)=z^{\ell}+c(t) .
$$

The correspondence $t \mapsto c(t)$ obeys the following properties:

- $c(0)=\tau$, 
- $t \mapsto c(t)$ is injective.

Proof. Assume that $c\left(t_{1}\right)=c\left(t_{2}\right)=c$ while $t_{1} \neq t_{2}$. Then $H \circ f_{\tau}=f_{\tau} \circ H$ where $H=\left(h^{t_{2}}\right)^{-1} \circ h^{t_{1}}$. We know that $H$ is quasi-conformal, $H$ is conformal off $J_{\tau}$, and $H(z) / z \rightarrow 1$ as $z \rightarrow \infty$. passing to the Bottcher coordinate of $f_{\tau}$ at infinity, we see that actually $H$ is the identity map off $J_{\tau}$, hence, everywhere. Then $h^{t_{1}}=h^{t_{2}}$ and $t_{1}=t_{2}$, a contradiction.

- $t \in \mathbf{D} \mapsto c(t) \in W$ is surjective. This follows from Proposition 4 .

- $c(t)$ is holomorphic in $t$. Indeed, $c(t)=f_{c(t)}(0)=h^{t} \circ f_{\tau} \circ\left(h^{t}\right)^{-1}(0)=h^{t}(\tau)$ is holomorphic in $t$, for fixed $\tau$, by the Ahlfors-Bers theorem on the dependence on parameters.

Thus we can put $R_{\tau}(t)=c(t)$.

\subsection{The conformal radius}

Now we calculate the conformal radius of $W$ at the point $\tau$. In other words, we need to find $\left|c^{\prime}(0)\right|$.

\section{Theorem 5}

$$
c^{\prime}(0)=-\frac{1}{\pi} \int \frac{\mu(z)}{z-\tau} d \sigma_{z}=-\frac{\ell}{\pi} \int z^{\ell-2} \mu(z) d \sigma_{z}
$$

Proof. Since $c(t)=h^{t}(\tau)$, we find

$$
c^{\prime}(0)=\left.\frac{\partial h^{t}}{\partial t}\right|_{t=0}(\tau)
$$

We apply general theory of quasi-conformal mappings [1] and particularly the proof of Th. 1 of [1], Ch. V. One can write:

$$
h^{t}(z)=P\left(\mu_{t}+\mu_{t} H_{t}\right)(z)
$$

where:

$P: L_{p} \rightarrow C(\mathbf{C}), p>2$, is the integral operator

$$
P g(z)=-\frac{1}{\pi} \int g(w)\left[\frac{1}{w-z}-\frac{1}{w}\right] d \sigma_{w}
$$

$H_{t}=R \mu_{t}+R \mu_{t} R \mu_{t}+R \mu_{t} R \mu_{t} R \mu_{t}+\ldots$, where, in turn, $R$ is a linear operator in $L_{p}$ (an extension of an integral singular operator, see [1]). Given $t,|t|<1$, the number $p>2$ can be chosen so that the $L_{p}$-norm of the operator $g \mapsto \mu_{t} R g$ is less 
than 1. Therefore, the series in the definition of $H_{t}$ converges in $L_{p}$ absolutely. Now, since $\mu_{t}=t \mu$ and $R$ is linear, $\mu_{t}+\mu_{t} H_{t}=t \mu+t^{2} B(t, z)$, where

$$
B(t, z)=R \mu+t R \mu R \mu+t^{2} R \mu R \mu R \mu+\ldots
$$

is bounded in $L_{p}$ uniformly on $t$ from a neighborhood of $t=0$. Thus,

$$
h^{t}(z)=z+t(P \mu)(z)+t^{2}(P B)(t, z) .
$$

Since $P \mu$ and $P B$ are continuous in $z$ and $t$, we finally get:

$$
\left.\frac{\partial h^{t}}{\partial t}\right|_{t=0}(\tau)=(P \mu)(z)=-\frac{1}{\pi} \int \mu(w)\left[\frac{1}{w-\tau}-\frac{1}{w}\right] d \sigma_{w} .
$$

Now, since $\mu$ is backward invariant Beltrami form on $J_{\tau}$, one can write

$$
\int \frac{\mu(w)}{w} d \sigma_{w}=\int \mu(w) T \frac{1}{w} d \sigma_{w}=0
$$

because

$$
T \frac{1}{z}=\lim _{a \rightarrow 0} \frac{1}{f^{\prime}(a)}\left[\frac{1}{z-f(a)}-\frac{1}{z-\tau}\right]=\lim _{a \rightarrow 0} \frac{a}{\ell(z-f(a))(z-\tau)}=0 .
$$

On the other hand, as we know after $(14), \frac{1}{z-\tau}=\left(T u_{*}\right)(z)$, where $u_{*}(z)=\ell z^{\ell-2}$. Using again the backward invariance of $\mu$, we are left with the formula

$$
\begin{aligned}
c^{\prime}(0)=\left.\frac{\partial h^{t}}{\partial t}\right|_{t=0}(\tau)= & -\frac{1}{\pi} \int \mu(w) \frac{1}{w-\tau} d \sigma_{w}=-\frac{1}{\pi} \int \mu(w) T u_{*}(w) d \sigma_{w} \\
& =-\ell \frac{1}{\pi} \int \mu(w) w^{\ell-2} d \sigma_{w}
\end{aligned}
$$

\section{$5 \quad$ Example 1}

In this section, we consider the Mandelbrot set $M_{2}$ (denoted by $M$ ). For $f=f_{c}$, denote by $\left(f^{k}\right)^{\prime}(z)$ the derivative of $f^{k}(z)$ w.r.t. $z, c_{k}(c)=f^{k}(0)$, and $c_{k}^{\prime}(c)=$ $\frac{d}{d c} c_{k}(c)$.

Let $\tau \in \partial M$ be a Misiurewicz value. Assume, for simplicity, that, for some $p>1, f_{\tau}^{p}(0)=a$, where $a$ is a repelling fixed point of $f_{\tau}$ with multiplier $\lambda_{\tau}$, $\left|\lambda_{\tau}\right|>1$. First, we construct the sequence of parameters $c_{n} \rightarrow c_{\tau}$ as follows. Fix a small neighborhood $Y$ of the point $a$, so that $\psi(Y) \subset Y$, for an inverse branch $\psi$ of $f_{\tau}$. Let $c_{-1}, \ldots, c_{-r}$ be a segment of a backward orbit of 0 by $f_{\tau}$, such that $c_{-r} \in Y, c_{-j} \notin Y, j \neq r$. Fix a small neighborhood $U_{\tau}$ of the parameter $\tau$. Then we 
have in $U_{\tau}$ analytic continuations $a(c), \lambda(c), \psi_{c}, c_{-j}(c)$, and sequence of analytic functions $g_{n}(c):=\psi_{c}^{n}\left(c_{-r}(c)\right)-f_{c}^{p}(0)$ in $U_{\tau}$, which converges, as $n \rightarrow \infty$, to a non-zero function $g(c):=a(c)-f_{c}^{p}(0)$. Since $g(\tau)=0$, there is a sequence $c_{n} \rightarrow \tau$ $\left(n>n_{0}\right)$, such that $g_{n}\left(c_{n}\right)=0$, i.e.

$$
f_{c_{n}}^{p_{n}}(0)=0
$$

where $p_{n}=p+n+r$.

Denote by $\Omega_{n}$ the component of the (bounded) Fatou set $F_{c_{n}}$ of $f_{c_{n}}$, which contains 0 . Let $\delta_{n}$ be the conformal radius of $\Omega_{n}$ at 0 , i.e. $\delta_{n}=\left|\phi_{n}^{\prime}(0)\right|$, where $\phi_{n}: \mathbf{D} \rightarrow \Omega_{n}, \phi_{n}(0)=0$, is the Bottcher coordinate of $f_{c_{n}}^{p_{n}}(0)=0$.

Remind $\mathbf{D}_{e}=\{z:|z|<e\}$.

Proposition 5 (jointly with F. Przytycki)

(1) There is a sequence $e_{n} \rightarrow 0$, such that

$$
\mathbf{D}_{\left(1-e_{n}\right) \delta_{n}} \subset \Omega_{n} \subset \mathbf{D}_{\left(1+e_{n}\right) \delta_{n}} .
$$

(2) There is a constant $L$, such that, for every $n$,

$$
\frac{\operatorname{area}\left(F_{c_{n}}\right)}{\operatorname{area}\left(\Omega_{n}\right)} \leq L
$$

$$
\frac{1}{L} \leq \frac{\operatorname{area}\left(\Omega_{n}\right)}{\operatorname{dist}\left(c_{n}, \partial M\right)} \leq L
$$

Proof. Let us consider a Yoccoz puzzle structure [10] for $f_{\tau}$ which starts with a periodic orbit other than the fixed point $a$. Now fix a level of the structure, such that:

each point $f_{\tau}^{k}(0), 0 \leq k \leq p$, is contained in a different (open) puzzle piece, $c_{-r} \in Y_{p}$ and $c_{-j} \notin Y_{p}, 0 \leq j<p$.

Let $Y_{0}, \ldots, Y_{p}$ be those pieces, so that $f_{\tau}^{k}(0) \in Y_{k}$. One can assume that $Y_{p}$ is the chosen above neighborhood $Y$ of $a$.

The same picture persists in the neighborhood $U_{\tau}$ of $\tau$. Denote by $Y_{k}(c)$ corresponding piece of $f_{c}$. Clearly, $Y_{k}(c)$ tends to $Y_{k}$ as $c \rightarrow \tau$.

Fix $f=f_{c_{n}}$. Let's pull back $Y_{0}\left(c_{n}\right)$ by $f$ along the orbit $f^{j}(0), 0 \leq j \leq p_{n}$. Let $Y^{n}\left(c_{n}\right)$ be the pullback: $0 \in Y^{n}\left(c_{n}\right)$ and $f^{p_{n}}\left(Y^{n}\left(c_{n}\right)\right)=Y_{0}\left(c_{n}\right)$. Then $f^{p_{n}}$ : $Y^{n}\left(c_{n}\right) \rightarrow Y_{0}\left(c_{n}\right)$ is a quadratic-like map [7]: it is conjugate by a quasi-conformal map $h_{n}$ to the map $z \mapsto z^{2}$, and $h_{n}: \mathbf{D} \rightarrow \Omega_{n}$ is conformal, i.e. $\left.h_{n}\right|_{\mathbf{D}}$ can be chosen to be equal to the Bottcher coordinate $\phi_{n}$. Since $\psi_{c_{n}}: Y_{p}\left(c_{n}\right) \rightarrow Y_{p}\left(c_{n}\right)$ is a contraction, $Y^{n}\left(c_{n}\right)$ shrink to the point 0 as $n \rightarrow \infty$. Therefore, the modulus of the fundamental annulus $Y_{p}\left(c_{n}\right) \backslash Y_{p}\left(c_{n}\right)$ tends to infinity, and the maximal dilatation of $h_{n}$ tends to zero as $n \rightarrow \infty$. It implies (1). 
Let's prove (2a). Let $V$ be a component of $F_{c_{n}}$ other than $\Omega_{n}$. Then $V$ contains a unique point $x$ such that $f_{c_{n}}^{k}(x)=0$, for some $k>0$. The branch $f_{c_{n}}^{-k}: \Omega_{n} \rightarrow V$ extends univalently to the neighborhood $Y_{0}\left(c_{n}\right)$. Hence, the distortion of all these inverse branches are uniformly bounded, in fact, tend to zero as $n \rightarrow \infty$ (because $\Omega_{n}$ shrink to 0 while $Y_{0}\left(c_{n}\right)$ is roughly fixed). Therefore,

$$
\operatorname{area}\left(F_{c_{n}}\right) \sim \operatorname{area}\left(\Omega_{n}\right) \cdot L_{n}
$$

where

$$
L_{n}=\sum_{n \geq 0} \sum_{f_{c_{n}}^{n}(x)=0} \frac{1}{\left|\left(f_{c_{n}}^{n}\right)^{\prime}(x)\right|^{2}} .
$$

Thus to prove (2a) we show that the sequence of $L_{n}$ is bounded from above. Since a definite neighborhood $K$ of $Y_{0}$ (and, by continuity, all $Y_{0}\left(c_{n}\right)$ ) contains no iterates of the critical point 0 , any branch $f_{c_{n}}^{-k}: \Omega_{n} \rightarrow V$ as above extends univalently to the neighborhood $K$. It follows that $L_{n}$ is comparable to the number $\frac{L_{n}^{\prime}}{\operatorname{area}\left(Y_{0}\left(c_{n}\right)\right)}$, where

$$
L_{n}^{\prime}=\sum_{k \geq 0} \sum_{f_{c_{n}}^{-k}} \operatorname{area}\left(f^{-k}\left(Y_{0}\left(c_{n}\right)\right),\right.
$$

and $f_{c_{n}}^{-k}$ runs over the all univalent branches defined on the neighborhood $K$. Since area $\left(Y_{0}\left(c_{n}\right)\right)$ is away from zero, $L_{n}$ is comparable to $L_{n}^{\prime}$. Thus we need to prove that the sum of the areas of all univalent preimages $\{Y\}$ of $Y_{0}\left(c_{n}\right)$ by the iterates of $f_{c_{n}}$ is bounded uniformly in $n$. All such preimages are ordered by inclusion. If $Y$ is such a preimage, its level is defined to be the number of other univalent preimages $Y^{\prime}$ which contain $Y$ and so that the next one contains the previous one. Sum of the areas of the preimages of the zero level is uniformly (in $n$ ) bounded because they are disjoint. Consider the preimages of the first level. By the bounded distortion, it is enough to consider them in $Y_{0}\left(c_{n}\right)$. Thus we get a map $R$ from the union of the preimages $V_{1} \subset Y_{0}\left(c_{n}\right)$ of the first level onto $Y_{0}\left(c_{n}\right)$. Since a definite part of $Y_{0}\left(c_{n}\right)$ (close to an equipotential in the boundary of $Y_{0}\left(c_{n}\right)$ ) never returns to it by the iterates of $f_{c_{n}}$, there is a constant $\alpha>0$ independent on $n$, such that the sum of the areas of the preimages $V_{1} \subset Y_{0}\left(c_{n}\right)$ of the first level is less than area $\left(Y_{0}\left(c_{n}\right)\right)(1-\alpha)$. Now, the preimages $V_{2} \subset Y_{0}\left(c_{n}\right)$ of the second level are preimages of $V_{1}$ by $R$. Because of the bounded distortion, the sum of the areas of $V_{2} \subset Y_{0}\left(c_{n}\right)$ is less than area $\left(Y_{0}\left(c_{n}\right)\right)\left(1-\alpha^{\prime}\right)^{2}$, where $\alpha^{\prime}<\alpha$ is independent on $n$. Continue this prosess, we see that the sum of the areas of the preimages $V_{k} \subset Y_{0}\left(c_{n}\right)$ of the level $k$ is less than area $\left(Y_{0}\left(c_{n}\right)\right)\left(1-\alpha^{\prime}\right)^{k}$. Thus $L_{n}^{\prime}$ are uniformly bounded, and the proof of (2a) is finished.

To prove (2b), let's calculate the conformal radius $\delta_{n}$. Differentiating twice the equation $f_{c_{n}}^{p_{n}}\left(\phi_{n}(w)\right)=\phi_{n}\left(w^{2}\right)$ at $w=0$, we get

$$
\delta_{n}=\frac{2}{\left|\left(f_{c_{n}}^{p_{n}}\right)^{\prime \prime}(0)\right|}=\frac{1}{\left|\left(f_{c_{n}}^{p_{n}-1}\right)^{\prime}\left(c_{n}\right)\right|} .
$$


On the other hand, $c_{n}$ is a center of a component $W_{n}$ of the interior of $M$, and the conformal radius $r_{n}$ of $W_{n}$ at $c_{n}$ is equal to (cf. Subsection 2.2)

$r_{n}=\left.\left|\left(f_{c_{n}}^{p_{n}-1}\right)^{\prime}\left(c_{n}\right) \cdot \frac{d}{d c}\right|{ }_{c=c_{n}} f_{c_{n}}^{p_{n}}(0)\right|^{-1}=\left|\left[\left(f_{c_{n}}^{p_{n}-1}\right)^{\prime}\left(c_{n}\right)\right]^{2} \cdot S_{p_{n}}\right|^{-1}=\delta_{n}^{2} \cdot\left|S_{p_{n}}\right|^{-1} \sim \frac{1}{\pi} \mathbf{a r e a}\left(\Omega_{n}\right)\left|S_{p_{n}}\right|^{-1}$,

where

$$
S_{p_{n}}=\sum_{k=0}^{p_{n}-1} \frac{1}{\left(f_{c_{n}}^{k}\right)^{\prime}\left(c_{n}\right)} .
$$

We complete the proof if we show that $S_{p_{n}}$ tend to a non-zero number. Let's prove that $S_{p_{n}}$ tend to $S_{\tau}$, where

$$
S_{\tau}=\sum_{k=0}^{\infty} \frac{1}{\left(f_{\tau}^{k}\right)^{\prime}(\tau)}
$$

By [18], [11], $S_{\tau} \neq 0$.

Let us divide the sum $S_{p_{n}}$ into 3 parts: $\sum_{n}^{1}=\sum_{k=0}^{p-1}, \sum_{n}^{2}=\sum_{k=p}^{p+n-1}$, and $\sum_{n}^{3}=\sum_{k=p+n}^{p+n+r-1}$. Obviously, as $n \rightarrow \infty, \sum_{n}^{1} \rightarrow \sum_{k=0}^{p-1} 1 /\left(f_{\tau}^{k}\right)^{\prime}(\tau)$. The sum $\sum_{n}^{3}$ contains a fixed number of summands, and each of them tends (as $n \rightarrow \infty$ ) to zero (because $\left|\left(f_{c_{n}}^{p_{n}-1}\right)^{\prime}\left(c_{n}\right)\right|=1 / \delta_{n} \rightarrow 0$ ), hence, $\sum_{n}^{3} \rightarrow 0$. Let us prove that $\sum_{n}^{2} \rightarrow \sum_{k=p}^{\infty} 1 /\left(f_{\tau}^{k}\right)^{\prime}(\tau)$. Denote $z_{n}=f_{c_{n}}^{p+n-1}\left(c_{n}\right)$. as $n \rightarrow \infty$. One writes:

$$
\sum_{k=p}^{p+n-1} \frac{1}{\left(f_{c_{n}}^{k}\right)^{\prime}\left(c_{n}\right)}=\frac{1}{\left(f_{c_{n}}^{p}\right)^{\prime}\left(c_{n}\right)} \sum_{j=0}^{n-1}\left(\psi_{c_{n}}^{j}\right)^{\prime}\left(\psi_{c_{n}}^{n-j-1}\left(z_{n}\right)\right) .
$$

Now, for $c \in U_{\tau}$, by the Konigs Linearization Theorem, $\psi_{c} \circ K_{c}(w)=K_{c}\left(w / \lambda_{c}\right)$, where $K_{c}$ is holomorphic at zero, $K(0)=a(c), K^{\prime}(0)=1$. Moreover, $K_{c}(z)$ is holomorphic in $(w, c)$ for $c \in U_{\tau},|w|<\epsilon$, and therefore $1 / K_{c}(w)=1+b_{1}(c) w+$ $b_{2}(c) w^{2}+\ldots$, where all $b_{k}(c)$ are holomorphic in $U_{\tau}$. Since $z_{n}=c_{-r}\left(c_{n}\right) \rightarrow c_{-r}$, one can assume that $z_{n}=K_{c_{n}}\left(w_{n}\right)$, where $w_{n} \rightarrow w_{*} \neq 0$. Then

$$
\left(\psi_{c_{n}}^{j}\right)^{\prime}\left(\psi_{c_{n}}^{n-j-1}\left(z_{n}\right)\right)=K_{c_{n}}^{\prime}\left(\left(\lambda\left(c_{n}\right)\right)^{-(n-1)}\right) \frac{\lambda\left(c_{n}\right)^{-j}}{K_{c_{n}}^{\prime}\left(w_{n} \lambda\left(c_{n}\right)^{-(n-j-1)}\right)},
$$

and

$$
\begin{gathered}
\sum_{j=0}^{n-1}\left(\psi_{c_{n}}^{j}\right)^{\prime}\left(\psi_{c_{n}}^{n-j-1}\left(z_{n}\right)\right)= \\
\left(1+O\left(|\lambda|^{-n}\right)\right) \cdot \sum_{j=0}^{n-1} \lambda\left(c_{n}\right)^{-j}\left(1+a_{1}\left(c_{n}\right) w_{n} \lambda\left(c_{n}\right)^{-(n-j-1)}+a_{2}\left(c_{n}\right)\left(w_{n} \lambda\left(c_{n}\right)^{-(n-j-1)}\right)^{2}+\ldots\right) \sim \\
\sum_{j=0}^{n-1} \lambda\left(c_{n}\right)^{-j} \sim \sum_{j=0}^{n-1} \lambda^{-j} .
\end{gathered}
$$




\section{On parabolic maps.}

Here we consider a quadratic map $f: z \mapsto z^{2}+c$ with a neutral parabolic periodic orbit $O=\left\{a_{1}, \ldots, a_{p}\right\}$ of exact period $p$. As usual, $\rho$ is the multiplier of $O$.

A formula for $1 / \rho^{\prime}$. We consider here the case $\rho \neq 1$ (for $\rho=1$, see below). Then the derivative $\rho^{\prime}$ of $\rho$ w.r.t. $c$ exists. By Theorem $3, \rho / \rho^{\prime}=2 \sum_{n>0} \sum_{f^{n}(w)=z}\left[\left(f^{n}\right)^{\prime}(w)\right]^{-2} / A(z)$, and one can try to pass to the limit as $z \rightarrow O$ using local dynamics. We make use of Theorem 2 instead. So, let $q>1$ be the minimal integer such that $\rho^{q}=1$. Denote $a=a_{0}=a_{p} \in O$. To state the result, we need the following notations.

- Complex number $K_{a}$ is defined by the expension

$$
f^{q}(z)=z+K_{a} \cdot(z-a)^{q+1}+O|z-a|^{q+2} .
$$

$K_{a} \neq 0$ because $f$ has a unique critical point.

- Function $R(z)$ defined in a small enough neighborhood of $a$ as follows. Given $j=0,1, \ldots, p-1$, denote by $f_{a_{j}}^{-1}$ the branch of $f^{-1}$ sending $a_{j}$ to $-a_{j-1}$ $\left(a_{-1}:=a_{p-1}\right)$. Then let

$$
R_{j}(z)=\sum_{f^{-n}}\left(\left(f^{-n} \circ f_{a_{j}}^{-1}\right)^{\prime}(z)\right)^{2}
$$

where $f^{-n}, n=0,1,2, \ldots$, run over all branches and all $n$ from a neighborhood of $-a_{j-1} ; R_{j}$ is defined and holomorphic in a neighborhood of $a_{j}$. Denote by $f_{0}^{-j}$ the branch of $f^{j}$ sending $a_{p}$ to $a_{j}, j=0,1, \ldots, p-1, p$. In particular, $f_{0}^{-p}$ maps $a_{p}$ to itself. At last, define

$$
R(z)=\sum_{j=0}^{p-1} \sum_{k=0}^{q-1} R_{j}\left(f_{0}^{-j} \circ f_{0}^{-p k}(z)\right) \cdot\left[\left(f_{0}^{-j} \circ f_{0}^{-p k}\right)^{\prime}(z)\right]^{2} .
$$

In other words, $R(z)=\sum\left[\left(f_{O}^{-n}\right)^{\prime}(z)\right]^{2}$ where $f_{O}^{-n}$ run over all those branches of $f^{-n}, n \geq 0$, which spend near the cycle $O$ at most $p q$ backward iterates, and then go away of $O$. Then $R$ is holomorphic at $a$. In fact, $R$ has at the point $a$ zero of order at least $q-2$. To see this, find a local holomorphic change of variable $h$, $h(0)=a$, such that $f_{0}^{-p}(h(u))=h(g(u))$ where $g(u)=\rho u+O|u|^{q+1}$, and use the following fact which is easy to check: If $\Psi$ is holomorphic at 0 , then

$$
\sum_{k=0}^{q-1} \Psi\left(g^{k}(u)\right)\left[\left(g^{k}\right)^{\prime}(u)\right]^{2}=q \Psi_{q-2} u^{q-2}+O|u|^{q},
$$

where $\Psi(u)=\sum_{n \geq 0} \Psi_{n} u^{n}$ is the power series. Thus there exists a complex number $P(a)$, such that, as $z \rightarrow a$,

$$
R(z) \sim P(a) \cdot(z-a)^{q-2} .
$$




\section{Theorem 6}

$$
\frac{1}{\rho^{\prime}}=\frac{1}{\rho} \frac{P(a)}{q K_{a}} .
$$

Proof. Let $A(z)$ be the function associated with the periodic orbit $O$. To apply Theorem 2, Part $\left(a^{*}\right)$, we need some preparations. Let $L$ be an attracting petal of $f^{p}$ at the point $a$, and let $\Phi:\{w: \operatorname{Re}(w)>M\} \rightarrow L$ be the Fatou coordinate, i.e. a holomorphic isomorphism, such that $f^{p q}(\Phi(w))=\Phi(w+1)$. It is known, that, as $w \rightarrow \infty, \Phi(w) \sim a+\frac{B}{w^{1 / q}}$, where the number $B$ is such that $B^{q}=-1 /\left(q K_{a}\right)$. Given a real number $m>M+1$, choose the fundamental region $C(m)$ as the image of vertical strip $\Pi(m): C(m)=\Phi(\Pi(m))$, where $\Pi(m)=\{w: m-1<R e(w)<m\}$. Now let $\mu(z)$ be any function from $L_{\infty}(C(m)),|\mu(z)| \leq 1$ a.e. $z \in C(m)$. It is easy to see that one can spread it out backward as well as forward and get an extension (denoted again by $\mu$ ) of $\mu$ from $C(m)$ to the whole (bounded) Fatou set $F$ of $f$ (i.e. $F$ is the basin of attraction of $O$ ), so that $\mu(f(z))=\mu(z)\left[f^{\prime}(z) /\left|f^{\prime}(z)\right|\right]^{2}$ a.e. in $F$.

\section{Fact 6.1}

$$
\int_{F} \mu(z) d \sigma_{z}=0
$$

This follows, for example, from Theorem 2, Part $\left(a^{*}\right)$. Indeed, it gives us that

$$
\int_{C(m)} \mu(z) A(z) d \sigma_{z}=\frac{2 \rho^{\prime}}{\rho} \int_{V(m)} \mu(z) d \sigma(z),
$$

where $V(m)=\cup_{n>0} f^{-n}(C(m))$. As $m \rightarrow+\infty, V(m)$ tends to $F$, so that it is enough to show that $\int_{C(m)} \mu(z) A(z) d \sigma_{z} \rightarrow 0$ as $m \rightarrow \infty$. As $w \rightarrow \infty(\operatorname{Re}(w)>M)$,

$$
A(\Phi(w))\left(\Phi^{\prime}(w)\right)^{2} \sim \frac{\left(\Phi^{\prime}(w)\right)^{2}}{(\Phi(w)-a)^{2}} \sim \frac{B^{2}}{q^{2} w^{2+2 / q}} \frac{1}{\left(B / w^{1 / q}\right)^{2}}=\frac{1}{q^{2} w^{2}} .
$$

It follows, as $m \rightarrow \infty$,

$$
\int_{\Pi(m)}|A(\Phi(w))| \cdot\left|\left(\Phi^{\prime}(w)\right)^{2}\right| d \sigma_{w} \sim \frac{1}{q^{2}} \int_{\Pi(m)} \frac{d \sigma_{w}}{|w|^{2}} \rightarrow 0 .
$$

Since $\left|\int_{C(m)} \mu(z) A(z) d \sigma_{z}\right| \leq \int_{C(m)}|A(z)| d \sigma_{z}=\int_{\Pi(m)}|A(\Phi(w))| \cdot\left|\left(\Phi^{\prime}(w)\right)^{2}\right| d \sigma_{w}$, it proves the fact.

This fact and (28) give us:

$$
\int_{C(m)} \mu(z) A(z) d \sigma_{z}=-\frac{2 \rho^{\prime}}{\rho} \int_{F \backslash V(m)} \mu(z) d \sigma(z) .
$$

This is the main equation to analyse. Make the following choice of $\mu=\mu_{m}$ on $C(m)$. Namely, let

$$
Q(m)=\{w: m-1<\operatorname{Re}(w)<m,-1<\operatorname{Im}(w)<1\} .
$$

Now choose $\mu_{m}$ so that: 
- $\mu_{m}(\Phi(w))=0$ for all $w \in P(m) \backslash Q(m)$,

$$
\mu_{m}(\Phi(w))=\frac{\left(\Phi^{\prime}(w)\right)^{2}}{\left|\Phi^{\prime}(w)\right|^{2}}
$$

for all $w \in Q(m)$.

First, we calculate the asymptotics (as $m \rightarrow+\infty$ ) of the left-hand side of (29). By the choice of $\mu_{m}$,

$$
\int_{C(m)} \mu_{m}(z) A(z) d \sigma_{z}=\int_{Q(m)} A(\Phi(w)) \cdot\left(\Phi^{\prime}(w)\right)^{2} d \sigma_{w} \sim \frac{1}{q^{2}} \int_{Q(m)} \frac{d \sigma_{w}}{w^{2}} \sim \frac{2}{q^{2}} \frac{1}{m^{2}} .
$$

Let us look at the right-hand side of (29). Denote $B(m)=\cup_{k=1}^{\infty} f^{p q k}(C(m))=$ $\Phi(\{w: \operatorname{Re}(w)>m\})$. Now it is easy to understand that $F \backslash V(m)=C(m) \cup$ $\cup_{n \geq 0} f_{O}^{-n}(B(m))$ where $f_{O}^{-n}$ are exactly the barnches from the definition of the function $R$. It follows from the invariance equation for $\mu_{m}$,

$$
\int_{F \backslash V(m)} \mu_{m}(z) d \sigma_{z}=\int_{C(m)} \mu_{m} d \sigma+\int_{C(m)} \sum_{k=1}^{\infty} R\left(f^{p q k}(z)\right)\left[\left(f^{p q k}\right)^{\prime}(z)\right]^{2} \mu_{m}(z) d \sigma_{z} .
$$

By the choice of $\mu_{m}$, the latter integral is equal to

$$
\int_{Q(m)} \sum_{k=1}^{\infty} R(\Phi(w+k))\left[\Phi^{\prime}(w+k)\right]^{2} d \sigma_{w}=\int_{G(m)} R(\Phi(w))\left[\Phi^{\prime}(w)\right]^{2} d \sigma_{w}
$$

where $G(m)=\{w: \operatorname{Re}(w)>m,-1<\operatorname{Im}(w)<1\}$ is horizontal strip. As $w \rightarrow \infty$,

$$
R(\Phi(w))\left[\Phi^{\prime}(w)\right]^{2} \sim P(a)\left(\frac{B}{w^{1 / q}}\right)^{q-2} \frac{B^{2}}{q^{2} w^{2+2 / q}}=\frac{1}{q^{2}} \frac{P(a)}{\left(-q K_{a}\right)} \frac{1}{w^{3}} .
$$

But

$$
\int_{G(m)} \frac{d \sigma_{w}}{w^{3}} \sim \frac{1}{m^{2}}
$$

Since $\int_{C(m)} \mu_{m} d \sigma=o\left(1 / m^{2}\right)$, we finally get

$$
-\frac{2 \rho^{\prime}}{\rho} \int_{F \backslash V(m)} \mu(z) d \sigma(z) \sim-\frac{2 \rho^{\prime}}{\rho} \frac{1}{q^{2}} \frac{P(a)}{\left(-q K_{a}\right)} \frac{1}{m^{2}}=\frac{2 \rho^{\prime}}{\rho} \frac{1}{q^{2}} \frac{P(a)}{q K_{a}} \frac{1}{m^{2}} .
$$

Compare (30) with (31), we get the result. 
Cauchy transform of $\mu$. Let again $\mu \in L_{\infty}(F)$ be any invariant (backward and forward) Beltrami form of $f$ on $F$. Fact 6.1 asserts that $\int_{F} \mu d \sigma=0$. Consider the Cauchy transform of $\mu: \hat{\mu}(z)=\int_{F} d \sigma_{u} /(u-z)$, which is continuous in the plane and holomorphic off $K=F \cup J$. But $\hat{\mu}(c)=2 \int_{F} \mu d \sigma=0$. ¿From the functional equation for $\mu$, see [11], we then get that $\hat{\mu}(z)=0$ for every $z \notin K$, i.e. $\mu$ is "invisible" outside the filled-in Julia set.

Cusps. In fact, very similar analysis as in Theorem 6 can be done when $\rho=1$ (cf. Comment 5). Then, instead of $\rho^{\prime}$ (which equals to $\infty$ in this case), one should consider $E:=\lim (1-\rho(\hat{c})) \rho^{\prime}(\hat{c})$ when $\hat{c} \rightarrow c$ from, say, inside of corresponding hyperbolic component. Then $E \neq 0, \infty[6]$. The result is that

$$
\frac{1}{E}=\frac{\sum_{\{x, n\}}\left[\left(f^{n}\right)^{\prime}(x)\right]^{-2}}{3\left[\left(f^{p}\right)^{\prime \prime}(a)\right]^{2}},
$$

where $\{x, n\}$ are all different points $x$ and corresponding minimal $n$, such that $f^{n}(x)=a$.

Doublings. In conclusion, consider the case $\rho=-1$. Then $K_{a}=\left(S f^{p}\right)(a) / 3$, where $S$ denotes the Schwarzian derivative, and $P(a)=2 \sum_{\{x, n\}}\left[\left(f^{n}\right)^{\prime}(x)\right]^{-2}$, where $\{x, n\}$ are all different points $x$ and corresponding minimal $n$, such that $f^{n}(x)=a$. Thus

$$
\frac{1}{\rho^{\prime}}=-3 \frac{\sum_{\{x, n\}}\left[\left(f^{n}\right)^{\prime}(x)\right]^{-2}}{\sum_{k=0}^{p-1}(S f)\left(f^{k}(a)\right)\left[\left(f^{k}\right)^{\prime}(a)\right]^{2}} .
$$

\section{Generalizations to polynomial-like maps.}

Main results are generalized to polynbomial-like maps with single critical point. Here we do this for quadratic critical point.

Let $f: U \rightarrow W$ be a polynomial-like map [7], i.e. $U, W$ are topological discs, $U$ is compactly contained in $W$, and $f$ is a holomorphic branced covering map of $U$ on $W$. We assume that $f^{\prime}(x)=0$ iff $x=0$ and $f^{\prime \prime}(0) \neq 0$. One can assume additionally that $f^{\prime \prime \prime}(0)=0$ (for example, $f(z)=Q\left(z^{2}\right)$, for a holomorphic $Q$, $\left.Q^{\prime}(0) \neq 0\right)$. Denote by $K_{f}$ the filled-in Julia set of $f$, and $d\left(K_{f}\right)$ its Eucledian diameter.

Let us imbed $f$ in the following family of mappings:

$$
f_{t}(z)=f(z)+t \cdot \frac{f^{\prime}(z)}{z}
$$

There exists a neighborhood of $t=0$ such that there $f_{t}: U_{t} \rightarrow W_{t}$ is still a polynomial-like map with the single quadratic critical point at 0 and with $U_{t}$ and $W_{t}$ close to $U$ and $W$ resp. 
Let $P=\left\{a_{1}, \ldots, a_{p}\right\}$ be a periodic orbit of $f$ with exact period $p$ and multiplier $\rho \neq 1$. Then it has an analytic continuation to a periodic orbit $\left\{a_{1}(t), \ldots, a_{p}(t)\right\}$ of $f_{t}$, so that $\rho(t)=\left(f_{t}^{p}\right)^{\prime}\left(a_{j}(t)\right)$ is analytic atound $t=0$. Denote $\rho^{\prime}=\rho^{\prime}(0)$. Let us introduce a "total variation" along the orbit $P$ :

$$
V(P)=p \cdot|\rho|+\frac{d\left(K_{f}\right)}{|1-\rho|} \sum_{k=1}^{p}\left|\left(f^{p}\right)^{\prime \prime}\left(a_{k}\right)\right| .
$$

For example, let the orbit $P$ be superattracting,i.e. $\rho=0$. In this case

$$
V(P)=d\left(K_{f}\right) \sum_{k=1}^{p}\left|\left(f^{p}\right)^{\prime \prime}\left(a_{k}\right)\right|=d\left(K_{f}\right) \sum_{k=1}^{p} \frac{2}{r_{k}}
$$

where $r_{k}$ is the conformal radius of the component of the basin of $P$ which contains $a_{k}$, at the point $a_{k}$.

Theorem 1 is generalized to polynomial-like maps as follows:

Theorem 7 There exist an absolute constant $\hat{L}$ and a decreasing function $M$ : $(0, \infty) \rightarrow(0, \infty)$ so that $M(m)=O(\exp (-m))$ as $m \rightarrow \infty$, such that, for any polynomial-like map $f$ with the the modulus $m$ of the fundamental annulus $W \backslash$ $U$, and for any attracting periodic orbit $P$ of $f$ with multiplier $\rho$, the following inequality holds:

$$
\hat{L} \cdot(1-|\rho|) \leq\left|\rho^{\prime}\right| \cdot \operatorname{area}\left(K_{f}\right)+M(m) \cdot V(P) \cdot \frac{\operatorname{area}\left(K_{f}\right)}{d\left(K_{f}\right)^{2}} .
$$

Comment 8 The family of maps $f_{t}$ appears naturally in the proof of the theorem. It also has the following nice property: for every periodic orbit with multiplier $\rho \neq 0$, the quantity $\left|\rho^{\prime}\right| \cdot \operatorname{area}\left(K_{f}\right)$ is invariant under linear changes of variable.

Comment 9 The function $M(m)$ is expressed through the following data: let $\gamma$ be the core curve of the fundamental annulus, $R=\operatorname{dist}\left(f^{-1}(\gamma), K_{f}\right)$, and $l$ is the Eucledian lenght of $f^{-1}(\gamma)$. Then $M(m)$ is equal essentially to $\left(d\left(K_{f}\right) / l\right)^{2}$. $(l / 2 \pi R) \cdot\left(1+d\left(K_{f}\right) / l\right)$.

Comment 10 Theorem 7 gives as well a generalization of the Douady-HubbardSullivan Theorem to polynomial-like maps. Namely, if attracting periodic orbit $P$ of the polynomial-like map $f$ is such that

$$
\hat{L} \cdot(1-|\rho|)>M(m) \cdot V(P) \cdot \frac{\operatorname{area}\left(K_{f}\right)}{d\left(K_{f}\right)^{2}}
$$

then $\rho^{\prime} \neq 0$. 
The proof of Theorem 7 is similar to the one of Theorem 1. It is contained in the following statements. Let $P=\left\{a_{1}, \ldots, a_{p}\right\}$ be a periodic orbit of $f$ with exact period $p$ and multiplier $\rho \neq 1,0$. As usual, denote

$$
A(z)=A_{P}(z)=\sum_{k=1}^{p} \frac{1}{\left(z-a_{k}\right)^{2}}+\frac{1}{\rho(1-\rho)} \sum_{k=1}^{p} \frac{\left(f^{p}\right)^{\prime \prime}\left(a_{k}\right)}{z-a_{k}}
$$

Firstly, we have the following generalization of Theorem 2:

Theorem 8 For any periodic orbit $P$ of $f$ as above, the function

$$
h(z):=A(z)-(T A)(z)-\rho^{\prime}(T 1)(z)
$$

is holomorphic throughout the range $W$.

The proof begins as follows. If $H$ and $\phi$ are any two functions holomorphic in $W \backslash K_{f}$, and $\gamma$ is any contour enclosing $K_{f}$ such that $f^{-1}(\gamma)$ is "inside" $\gamma$, then a change of variable gives us:

$$
\frac{1}{2 \pi i} \int_{\gamma} H(z)[\phi(z)-(T \phi)(z)] d z=\frac{1}{2 \pi i} \int_{f^{-1}(\gamma)} \phi(z)\left[H(z)-\frac{H(f(z))}{f^{\prime}(z)}\right] d z
$$

Now we put $H$ to be any function holomorphic in $W$ and $\phi=A$. Then we use the Residue Theorem and the following two identities

$$
\frac{f^{\prime \prime}\left(a_{k}\right)}{\left(f^{\prime}\left(a_{k}\right)\right)^{2}}-\frac{1}{\rho(1-\rho)} \frac{\left(f^{p}\right)^{\prime \prime}\left(a_{k}\right)}{f^{\prime}\left(a_{k}\right)}=-\frac{\left(f^{p}\right)^{\prime \prime}\left(a_{k+1}\right)}{\rho(1-\rho)}
$$

and

$$
A(0)=-\frac{\rho^{\prime}}{\rho}
$$

which can be proved very similar to the proofs of analogous identities in Section 2. As a result we get that $\frac{1}{2 \pi i} \int_{f^{-1}(\gamma)} H(z) h(z) d z=0$ for every holomorphic function $H$ in $W$. The statement that $h$ is holomorphic in $W$ follows. Now we can use the Maximum Principal to estimate $h$ on the set $K_{f}$. At last, we repeat the proofs of Propositions 2 and 3, and get the result.

\section{References}

[1] Ahlfors, L.:Lectures on quasiconformal mappings. Van Nostrand, 1966

[2] Carleson, L. \& Gamelin, T.: Complex dynamics. Springer-Verlag, 1993

[3] Coullet, P. \& Tresser, C.: Iteration d'endomorphismes et groupe de renormalisation, CRAS 287 A (1978) 
[4] Douady, A.: Systemes dynamiques holomorphes. Seminare Bourbaki, v. 198283, n. 599, Asterisque, 105-106, 1983, 39-63

[5] Douady, A. \& Hubbard, J.H.: Iteration des polynomes quadratiques complexes. C.R.A.S., t. 294 (1982), 123-126

[6] Douady, A. \& Hubbard, J.H.: Etude dynamique des polynomes complexes. Pub. Math. d'Orsay, 84-02 (1984), 84-04 (1985)

[7] Douady, A. \& Hubbard, J.H.: On the dynamics of polynomial-like maps. Ann. Sc. Ec. Norm. Sup., v. 18 (1985), 287-343

[8] Epstein, A.: Infinitesimal Thurston rigidity and the Fatou-Shishikura inequality, preprint Stony Brook, 1999.

[9] Feigenbaum, M.: Qualitative universality for a class of non-linear transformations, J. Stat. Phys. 19 (1978), 25-52

[10] Hubbard, J. H.:Local connectivity of Julia sets and bifurcation loci: three theorems of J.-C. Yoccoz. In: "Topological Methods in Modern Mathematics." Publish or perish, 1993

[11] Levin, G.: On an analytic approach to the Fatou conjecture. To appear in Fundamenta Mathematicae

[12] Levin, G., Sodin, M. \& Yuditski, P.: A Ruelle operator for a real Julia set. Comm. Math. Phys. 141 (1991), 119-132

[13] Lyubich, M.:Feigenbaum-Coullet-Tresser universality and Milnor's hairiness conjecture. Annals of Math. v. 149 (1999), 319-420

[14] Mane, R., Sad, P. \& Sullivan, D.: On the dynamics of rational maps. Ann. Sci. Ec. Norm. Sup. 16 (1983), 193-217

[15] Makienko, P.: Remarks on Ruelle operator and invariant line field problem. Part I: preprint FIM, 25, Zurich, July 2000. Part II: preprint, 2001.

[16] McMullen, C.: Complex dynamics and renormalization, Ann. of Math. Studies 135, Princeton University Press (1994)

[17] McMullen, C.: Renormalization and 3-manifolds which fiber over the circle, Ann. of Math. Studies 142, Princeton University Press (1996)

[18] Rivera-Letelier, J.:On the continuity of Hausdorff dimension of Julia sets and similarity between the Mandelbrot set and Julia sets. Fundamenta Mathematicae, v. 170 (2001), n. 3, 287-317

[19] Sullivan, D.:Bounds, quadratic differentials and renormalization conjecture. In: Mathematica into twenty-firts century, AMS Centennial publications (1991)

[20] Tsujii, M.:A simple proof for monotonicity of entropy in the quadratic family. Erg. Th. Dyn. Syst. 20 (2000), n. 3, 925-933 